\title{
Das rezeptionsgrammatische Modell am Beispiel der erweiterten Substantivphrase in ausgewählten deutschen Wirtschaftstexten
}

\begin{abstract}
It is a well-known fact that many students of German have difficulties reading and thereby understanding LSP texts written in German. Why? This is the kind of question that is normally answered within a cognitive framework. In this article, however, the point of departure will be syntactic. The perspective will be receptive (and not productive or neutral as is the case with traditional German grammar and generative grammar, respectively). The signals on the text surface will be given high priority due to the receptive perspective. The main purpose of the article will be to present and discuss a syntactic model for analysing German NPs in economic texts from a receptive perspective. The model is based on four description principles: the "Phrase", the "Rahmen", the "Feld" and finally the "Ketten" principles. The "Ketten" principle is the most characteristic for the NP model presented here as it offers an alternative way of looking at post-modifiers. In the article it will then be argued that the model is useful for description and that the results allow us to make hypotheses as to answering the question above.
\end{abstract}

\section{Einleitung1}

Generell gesehen liegen bereits dutzende Arbeiten syntaktischer Art zum Thema Substantivphrase vor. Hier sei nur auf wenige auf der generativen Grammatik beruhende hingewiesen wie Bierwisch 1961, Kolde 1985, Vater 1986, 1992, Haider 1988, 1992, Bhatt 1990, Löbel 1990, Zimmermann 1991. Hervorzuheben ist dabei der Artikel von Gallmann/Lindauer (1994), in dem ein Überblick über den generativen

1 Für wertvolle Hinweise und Anregungen möchte ich mich besonders bei Prof. Dr. Wolfgang Koch (Wirtschaftsuniversität Aarhus), aber auch bei den beiden Beurteilern der Zeitschrift Hermes bedanken.

* Marianne Grove Ditlevsen Tysk Institut

Handelshфjskolen i Århus

Fuglesangs Allé 4

DK-8210 Arhus V

Hermes, Journal of Linguistics no. 22 - 1999 
Ansatz zur Beschreibung der Substantivphrase vermittelt wird. Es sei hier auch auf nur zwei auf in grammatischer Hinsicht eher traditionelleren Perspektiven beruhende Arbeiten älteren Datums hingewiesen, und zwar Sommerfeldt 1968 und Teubert 1979.

Innerhalb der Fachsprachenlinguistik ist die Situation fast dieselbe, zumal eben die Substantivphrase öfters als Fachtextmerkmal herangezogen wird. So kann es nicht überraschen, daß die Substantivphrase in fachsprachenlinguistischen Arbeiten oft thematisiert wird (z.B. Benes 1981, Kretzenbacher 1991).

Vor diesem Hintergrund ist es angebracht, sich die Frage zu stellen, womit nun erneute Ausführungen zur Substantivphrase wie die vorliegenden zum Thema beitragen können. In erster Linie unterscheidet sich die hier vorgestellte Arbeit von den obenerwähnten grundsätzlich dadurch, daß sie nicht Sprecher-/Hörer-neutral gestaltet ist, sondern die rezeptive Perspektive als Ausgangspunkt hat. Im vorliegenden Artikel wird nämlich ein Modell zur grammatischen Beschreibung der aktuell vorkommenden Substantivphrasen aus rezeptiver Perspektive, das sogenannte rezeptionsgrammatische Modell, erstellt, diskutiert und angewandt. Eine solche Einarbeitung einer rezeptiven Perspektive in die Grammatik und grammatische Beschreibungen findet sich nur bei wenigen Arbeiten überhaupt. So sind mir, was die deutsche Sprache betrifft, nur die Grammatiken (die grammatischen Beschreibungen) Rall/ Engel/Rall 1985, Fabricius-Hansen/Ahlgren 1986 und Heringer 1989 sowie die Arbeit, die in Fabricius-Hansen/Solfjeld 1994 präsentiert wird, bekannt. ${ }^{2}$ Dabei ist das hier vorgestellte rezeptionsgrammatische Modell der erste Versuch, ein Modell zur Beschreibung der gesamten Substantivphrase aus rezeptiver Perspektive zu entwickeln. Getestet wird das Modell durch eine Beschreibung von Substantivphrasen in Fachtexten der Wirtschaft. Und somit leistet dieser Artikel auch einen Beitrag zur Fachsprachenlinguistik, zumal die meisten Arbeiten der Fachsprachenlinguistik sich bekanntlich mit anderen Fachsprachen wie die des Rechts oder der Technik befassen.

Der Wunsch, die rezeptive Perspektive in eine syntaktische Beschreibung einzubauen, ist im Unterricht entstanden. Als Dozentin an einer dänischen Wirtschaftsuniversität bin ich nämlich oft auf das Problem gestoßen, daß es dänischsprachigen Studenten, die Deutsch als

2 Es wird in dem Zusammenhang von Diplomarbeiten abgesehen. 
Fremdsprache studieren, oft schwerfällt, selbst weniger komplizierte deutschsprachige Fachtexte zu lesen und somit auch zu verstehen. Dies ist nicht nur für die meisten dänischen Dozenten ein bekanntes Problem, sondern überhaupt für skandinavische Dozenten. So heißt es z.B. in Fabricius-Hansen/Solfjeld 1994:

"Norwegische Studenten haben wenigstens in den ersten Semestern ihres Deutschstudiums z.T. erhebliche Schwierigkeiten beim Lesen deutscher Sachprosatexte. [...] Es fragt sich, welche Aspekte der deutschen Gebrauchsprosa Norwegern beim Lesen, im rezeptiven Bereich, besondere Probleme bieten - und warum?"

(Fabricius-Hansen/Solfjeld 1994: 2)

Diese Überlegungen, die auch für dänische Verhältnisse von Relevanz sind, bildeten den Ausgangspunkt für die Analysearbeit, die in Ditlevsen 1996 ausführlich behandelt wird und die hier in Auszügen präsentiert wird, zumal es Hauptziel der Analysen gewesen ist, eine Hypothese darüber aufzustellen, inwieweit morpho-syntaktische Strukturen der Substantivphrase zu den rezeptiven Schwierigkeiten der dänischsprachigen Deutschstudenten beitragen könnten.

Der Artikel teilt sich in zwei logische Hauptabschnitte, und zwar in einen Abschnitt 1, in dem das rezeptionsgrammatische Modell vorgestellt und diskutiert wird, und einen Abschnitt 2, in dem das rezeptionsgrammatische Modell zur Analyse von Substantivphrasen in Fachtexten der Wirtschaft angewandt wird. Den Kern im Abschnitt 2 bilden ausgewählte Ergebnisse. Im dritten und letzten Abschnitt finden sich Konklusion und Perspektivierung.

\section{Das rezeptionsgrammatische Modell}

Den theoretischen Rahmen des Modells bildet die traditionelle Grammatik, wie sie beispielsweise von der Duden-Grammatik (Duden Band 4, 1995) und Helbig/Buscha 1996 vertreten wird; jedoch wird sie - wie oben schon erwähnt - durch die rezeptive Perspektive ergänzt, so wie es bei Fabricius-Hansen/Ahlgren 1986 und Heringer 1989, die als rezeptiv orientierte Beschreibungen zu betrachten sind, zu sehen ist.

Als Grundlage der rezeptiven Grammatik wird davon ausgegangen, daß die Grammatik nicht - wie von manchen Grammatikern angenommen - Sprecher-/Hörer-neutral ist, sondern daß es auch im grammatischen Bereich wichtig ist, zwischen aktiver und passiver Kompetenz zu unterscheiden, also zwischen Produktion und Rezeption, die sich 
gegenseitig unterstützen. Von einer rezeptiven Grammatik wird nach Fabricius-Hansen/Heringer (1988: 165f.) eine semasiologische Systembeschreibung im Gegensatz zur eher traditionellen onomasiologischen Beschreibung verlangt. Darüber hinaus gehören zu einer rezeptiven Grammatik erstens die Erforschung der Textverarbeitung, so wie es anhand neuerer kognitiver Theorien geleistet wird, bei denen der prozessuale Aspekt im Zentrum steht, und zweitens die Aufstellung von Rezeptionsstrategien, die als eigentliche Leseanweisungen ihren Niederschlag finden, so wie es beispielsweise bei Heringer (1989: 20), oder bei Fabricius-Hansen/Ahlgren (1986: 71), zu sehen ist.

Das für meine Analysen angewandte Modell wird nur der ersten Aufgabe gerecht, zumal es weder zur Erforschung der Textverarbeitung beiträgt noch Rezeptionsstrategien aufstellt. Das Analysemodell, das ich das rezeptionsgrammatische Modell nenne, ist lediglich ein Modell zur syntaktischen Beschreibung der gesamten Substantivphrase unter Berücksichtigung der rezeptiven Perspektive.

Das rezeptionsgrammatische Modell läßt sich als ein lineares Modell charakterisieren, weil die Textoberfläche (und somit die Topologie) eine große Rolle spielt. Dies ist darauf zurückzuführen, daß die lineare Folge von Wörtern und graphematischen Zeichen das einzig Konkrete ist, das dem Rezipienten bei der Rezeption zur Verfügung steht. Dabei ist dies übrigens auch der wesentlichste Grund, weshalb auf einen generativen Ansatz verzichtet wurde. Bei der syntaktischen Beschreibung ist es jedoch selbstverständlich unumgänglich, auch auf die hierarchischen Strukturen der Substantivphrasen Rücksicht zu nehmen. Das Modell baut auf die bekannten Prinzipien der Phrase, der Klammer und der Feldstruktur. Dabei ist der Begriff "Prinzip" in erster Linie als Beschreibungsprinzipien oder auch Beschreibungsinstrumente zu verstehen. Die oben geforderte rezeptive Perspektive für das rezeptionsgrammatische Modell zur Beschreibung der Substantivphrase kommt bei den drei Prinzipien nur durch das Klammerprinzip zum Ausdruck (vgl. unten). Ganz deutlich wird die rezeptive Perspektive erst durch die Einbeziehung des für das rezeptionsgrammatische Modell charakteristischsten Prinzips, des Kettenprinzips. Im folgenden wird zwecks Aufstellung des gesamten rezeptionsgrammatischen Modells näher auf die vier grundlegenden Prinzipien eingegangen. 


\subsection{Das Phrasenprinzip}

Das Phrasenprinzip, das als das übergeordnete sprachstrukturelle Prinzip zu bezeichnen ist, besagt ganz einfach, daß die hier zu beschreibende Wortgruppe als eine Phrase gilt, weil sie unabhängig vom Kontext beschrieben wird (im Gegensatz zu den Satzgliedern) (vgl. Engel 1988-1991: 22). Die Phrase ist eine Substantivphrase, zumal sie ein Substantiv als Kern hat. Der Kern wird mit Engel (1988-1991: 22), als das oberste Element der Phrase verstanden, von dem alle anderen Teile der Phrase abhängen, und der sich durch die Wegstreichprobe bestimmen läßt. Der Kern entspricht dem Kopf ("Head") der generativen Grammatik, in der der Begriff Kern als Synonym für Kopf auch zu finden ist, so z.B. bei Gallmann/Lindauer 1994.

Die Phrase wird in Abb. 1 durch $\int \ldots \int$ graphisch dargestellt, und der Kern durch K, im Beispiel 1 durch Hervorhebung. Die dem Kern voran- und nachgestellten Lücken geben an, daß - wie bekannt - dem Kern Attribute voran- oder auch nachgestellt sein können.

Abb. 1

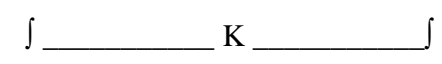

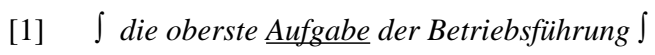

$(\text { de.c. } 1.1)^{3}$

\subsection{Das Klammerprinzip}

Das zweite Prinzip, das Klammerprinzip, ist psychologisch begründet, zumal ein intuitiv angenommener Spannungsbogen, wie er beispielsweise bei Boost 1964 beschrieben wird, die Grundlage des Prinzips bildet. Es wird angenommen, daß ein Teil der Substantivphrase als eine Klammer beschrieben werden kann, die von dem Artikelwort als linkem Klammerelement und dem Kern der Substantivphrase als rechtem Klammerelement gebildet wird. Das Klammerprinzip wird in Abb. 2 durch die eckigen Klammern graphisch dargestellt; dazu auch Beispiel 2. Das linke Klammerelement ist dabei das Artikelwort (AW), ${ }^{4}$ wie es bei Helbig/Buscha 1996 definiert wird, und das rechte Klammerelement ist der Kern $(\mathrm{K})$.

3 Die Quellenangabe verweist auf die für die Analysen benutzten Korpora.

4 Die in diesem Artikel verwendeten, nicht üblichen Abkürzungen sind alle im Anhang zu finden. 
Abb. 2

$$
\int\left[\mathrm{AW} \_\mathrm{K}\right]
$$

[2] $\int$ [die oberste Aufgabe] der Betriebsführung $]$

(de.c.1.1.)

Den Teil der Substantivphrase, der durch die Klammer sozusagen umrahmt wird einschließlich AW und K, nenne ich mit Fabricius-Hansen/ Solfjeld (1994: 49), den Substantivrahmen. Im Beispiel 2 ist der umklammerte Teil "die oberste Aufgabe" somit ein Substantivrahmen.

Während das Phrasenprinzip ein allgemein anerkanntes Prinzip der Sprachstruktur ist, so stellt die Annahme einer Nominalklammer ${ }^{5}$ innerhalb der Linguistik wenn nicht ein viel diskutiertes Thema, dann jedoch einen Bereich dar, über den viel diskutiert werden kann. Für die Annahme einer Nominalklammer spricht u.a., daß die Nominalklammer genau wie die Verbalklammer als Ausdruck eines intuitiv angenommenen Spannungsbogens betrachtet werden kann. ${ }^{6}$ Somit hat man (als Rezipient) durch den Artikel als klammeröffnendes Element "das Signal bekommen [...], auf etwas zu warten, zudem die Information, mit welchem Grad an Spezifizität oder Bekanntheit bzw. Neuheit man bei der zu erwartenden nominalen Entität zu rechnen habe, verbunden mit unterschiedlich genauen morphologischen Informationen (Genus, Numerus, Kasus)." (Eichinger 1992: 88). Wider die Annahme einer Nominalklammer sprechen vor allem syntaktisch begründete Einwände, die auf prinzipiellen Unterschieden zwischen der Nominalklammer und der Verbalklammer beruhen (Kolde 1985 und Eisenberg 1989). Darüber hinaus ist es fraglich, inwieweit es möglich ist - wie Eisenberg (1989: 28) vorführt -, den Sonderstatus des Artikels als klammeröffnenden Elements syntaktisch zu begründen, zumal beispielsweise nicht nur der Artikel, sondern auch das attributive Adjektiv unter Umständen die Genusmarkierung wahrnimmt. Der erste Einwand ist dadurch bedingt, daß Kolde und Eisenberg nur eine Verbalklammer für den Hauptsatz annehmen. Bekanntlich nehmen die meisten Linguisten jedoch auch eine Verbalklammer für den Nebensatz an. Was den zweiten Einwand betrifft, kann hervorgehoben werden, daß der Artikel in

5 Hierfür werden auch Begriffe wie "Nominalrahmen" (Kolde 1985) und "nominaler Rahmen" (Helbig/Buscha 1996) verwendet.

6 Ein solcher Vergleich zwischen dem Satz und der Substantivgruppe ist schon bei Admoni 1934 zu sehen. 
mindestens zweierlei Hinsicht zu recht einen Sonderstatus genießt: Erstens hängt die Form des Adjektivs jederzeit von An- / Abwesenheit und Form des Artikels ab (Beispiel 3 - 6), wogegen die Form des Artikels niemals von An- / Abwesenheit und Form des Adjektivs abhängen kann (Beispiel 7 - 9). Zweitens ist "die Aufgabe des Artikels eine Spezifitätsangabe [...] und die Aufgabe der von dem Rahmen umfangenen Elemente die Attribution" (Eichinger 1992: 89-90).

[3] das gute Beispiel

[4] ein gutes Beispiel

[5] alle guten Beispiele

[6] gute Beispiele

[7] das Beispiel

[8] ein Beispiel

[9] alle Beispiele

Entscheidend für die vorliegende Arbeit ist es dabei jedoch nicht, inwieweit sich die Annahme einer Nominalklammer syntaktisch begründen läßt, sondern inwieweit die Annahme einer Nominalklammer zur Beschreibung der Substantivphrase in Wirtschaftstexten aus rezeptiver Perspektive beitragen kann. Aus diesem Grund wird dem Spannungsbogen als gemeinsamem Nenner der Verbal- und Nominalklammer viel Wert beigemessen, zumal der (intuitiv angenommene) Spannungsbogen sowohl im Satz als auch in der Substantivphrase beim Rezipienten dieselbe Reaktion auslöst. Man vergleiche dazu die untenstehenden Zitate, wobei sich das Zitat aus Admoni 1934 auf die Verbalklammer bezieht, und das Zitat aus Weinrich 1993 auf die Nominalklammer.

"Da das Prädikatsnomen zum Verstehen des Satzes unentbehrlich ist, dabei zum Hörer (oder Leser) erst als allerletztes Satzglied gelangt, wird eine gewisse Spannung hervorgerufen, die so lange anhält, wie der Satz gesprochen (gelesen) wird. Dadurch wird die Aufmerksamkeit für die Aufnahme aller, selbst der nebensächlicheren Satzteile erheblich gesteigert." (Admoni 1934: 79)

"Ebenso wie die Verbalklammer erzeugt auch die Nominalklammer beim Hörer eine bestimmte Spannung. Der Hörer muß nämlich, wenn er die Nominalklammer als ganze verstehen will, alle Sprachzeichen, die im Klammerfeld zwischen dem Artikel als dem klammeröffnenden Element und dem Nomen als dem klammerschließenden Element stehen, so lange in seinem Kontextgedächtnis speichern, bis er das Nomen selber rezipiert hat." (Weinrich 1993: 356) 


\subsection{Das Feldprinzip}

Das dritte übergeordnete Prinzip, das Feldprinzip, ist topologischer Natur, zumal die angenommene Feldstruktur vor allem durch die Stellungsrestriktionen bzw. Stellungsgemeinsamkeiten der einzelnen Elemente bestimmt wird. Dem Feldbegriff werden innerhalb der Linguistik recht unterschiedliche Bedeutungen zugeschrieben, wie es beispielsweise schon bei V fisur 1977 zu sehen ist. Deswegen ist es wichtig an dieser Stelle zu explizitieren, daß der hier verwendete Feldbegriff zur Beschreibung der Abfolgeregularitäten (wie es bei Eisenberg (1989: 425) bezeichnet wird) innerhalb der Substantivphrase auf Drach 1940 zurückgeht, bei dem der Begriff für die Beschreibung des sogenannten deutschen Satzbauplans verwendet wurde. Der Bedarf an einem Satzbauplan war für Drach im Unterricht zu finden, zumal der Satzbauplan als Hilfsmittel im (Fremdsprachen-) Unterricht verstanden werden sollte. So heißt es u.a.:

"Beide, Deutsche wie Ausländer, brauchen nicht eine abstrakte Beschreibung allgemeiner syntaktischer Erscheinungen, sondern eine konkrete Anleitung zu zweckmäßigen Sprechhandlungen.”

(Drach 1940: 12)

"Dem Ausländer dient [das Leitbild einer mittleren Norm (= der Satzbauplan, mgd)] als Arbeitsanweisung. [...] Für den Deutschen liefert es Beboachtungshilfen, die Gestaltungsweise der Zeitsprache zu durchschauen.” (Drach 1940: 12)

Es ist wichtig daran festzuhalten, daß der Satzbauplan also nicht wissenschaftlich begründet war, sondern didaktisch, und daß er nicht der Beschreibung sprachstruktureller Elemente diente, sondern als Hilfsmittel. Dies ist u.a. wichtig, weil von Askedal behauptet wird, daß

"im Rahmen der [Feldkonzeption (eigentlich die Stellungsfelderkonzeption, mgd)] mit einer zusätzlichen Ebene der "Stellungsfelder" gearbeitet [wird], die zwischen Satz und Konstituenten des Satzes (Satzgliedern) anzusiedeln ist.” (Askedal 1986.a: 269)

Diese Ansicht erscheint nicht unmittelbar wohlbegründet $\mathrm{zu}$ sein, zumal wir es hier mit zwei völlig verschiedenen Betrachtungsweisen zu tun haben, und zwar bei den Ebenen des Satzes bzw. der Konstituenten mit einer syntaktischen, und bei der Ebene der Stellungsfelder mit einer topologischen.

Bevor auf die Felderstruktur innerhalb der Substantivphrase eingegangen wird, soll zunächst der Satzbauplan von Drach präsentiert wer- 
den, zumal er als Grundlage der Felderstruktur der Substantivphrase zu sehen ist. Den Ausgangspunkt für den Satzbauplan bei Drach bildet die Ansicht, daß das finite Prädikat "der standfeste Angelpunkt [ist], um den herum der Satz sich aufbaut und gliedert" (Drach 1940: 16). Das finite Prädikat steht somit in der Mitte, wie Drach die Stelle nennt. Für die Mitte hat sich der Begriff Mittelfeld inzwischen als Terminus dafür eingebürgert, wie es beispielsweise bei Duden-Grammatik (1995: 787) zu sehen ist. Die Elemente, die vor der Mitte stehen, befinden sich im Vorfeld, und die Elemente, die nach der Mitte stehen, im Nachfeld. Im Satzbauplan können allein den Elementen der Mitte, also den finiten Prädikaten, Gemeinsamkeiten zugeschrieben werden. Der Grundgedanke, der bei Drach zum ersten Mal zum Ausdruck kam, hat zur Weiterentwicklung des Satzbauplanes geführt. So sind beispielsweise zur Illustration in der Duden-Grammatik insgesamt vier Satzbaupläne zu finden, wobei für den Kernsatz, der dem Aussagehauptsatz entspricht, folgendes Schema, das insgesamt sechs sogenannte Stellungsfelder enthält, aufgestellt wird. Das finite Verb und der infinite Verbalkomplex machen zugleich die Satzklammer aus:

\begin{tabular}{|l|l|l|l|l|l|}
\hline $\begin{array}{l}\text { beiordnende } \\
\text { Konjunktionen }\end{array}$ & Vorfeld & finites Verb & Mittelfeld & $\begin{array}{l}\text { infiniter } \\
\text { Verbalkomplex }\end{array}$ & Nachfeld \\
\hline
\end{tabular}

(Duden-Grammatik 1995: 788)

Wie es bei manchen anderen ursprünglich satzbezogenen linguistischen Begriffen, wie z.B. dem oben behandelten Klammerprinzip, der Fall ist, ist die Einteilung einer Konstituente in Stellungsfelder auch auf die Substantivphrase übertragen worden. Eine solche Einteilung der Substantivphrase in Stellungsfelder ist u.a. bei Duden-Grammatik 1995, Askedal 1986, Sommerfeldt 1971 und Lauridsen/Poulsen 1995 zu sehen. Im Vergleich zum Satz läßt sich übergeordnet sagen, daß die Abfolgeregularitäten der Substantivphrase fester sind. Hier lassen sich im Gegensatz zum Satz somit sämtliche Felder anhand stellungsmäßiger / topologischer Gemeinsamkeiten beschreiben, wie es aus folgendem ersichtlich ist.

Im rezeptionsgrammatischen Modell konstituiert der Kern der Substantivphrase das rechte Klammerfeld (einfacher: das Kernfeld; KF). Vor dem Kernfeld befinden sich das linke Klammerfeld (einfacher: Artikelfeld; AF) und das Vorfeld (VF), wobei das Artikelfeld von den 
Artikelwörtern konstituiert wird, und das Vorfeld von den Adjektivund Partizipialattributen. Die Unterscheidung zwischen AF und VF ist im Prinzip eine logische Folge der Annahme einer Nominalklammer, wie sie oben präsentiert wurde. Das Feldprinzip wird in Abb. 3 sowie im Beispiel 10 durch | graphisch dargestellt.

Abb. 3

$$
\int[\mathrm{AF}|\mathrm{VF}| \mathrm{KF}] \ldots \int
$$

[10] $\int[$ das $\mid$ theoretische $\mid$ Modell $] .. . \int$

(de.c.1.3)

Im Artikelfeld finden wir auch die vorangestellten Substantive im Genitiv (Beispiel 11) und im Vorfeld die Substantive, die als sogenannte enge Appositionen fungieren (Beispiel 12).

[11] $\int\left[\right.$ Hoffmanns $|-|$ Subsprachentheorie ]... $\int$

[12] $\int[-\mid$ Professor $\mid$ Kuno $] \ldots \int$

Nach dem Kernfeld befinden sich das Schlußfeld (SF) und das Zusatzfeld (ZF) (s. Abb. 4), wobei das Schlußfeld von den postnuklearen substantivischen Attributen und das Zusatzfeld von den attributiven Nebensätzen und Infinitivkonstruktionen konstituiert wird. Im Schlußfeld kommen sowohl Substantivrahmen im Präpositionalkasus als auch Substantivrahmen im Genitiv in Frage (Beispiel 13 und 14). Im Zusatzfeld kommen attributive Relativsätze (Beispiel 15), Konjunktionalsätze (Beispiel 16) und attributive Infinitivkonstruktionen (Beispiel 17) vor.

Abb. 4

$$
\int[\ldots \mathrm{KF}]|\mathrm{SF}| \mathrm{ZF} \int
$$

[13] $\int[\ldots$ Bestandsveränderungen $] \mid$ an unfertigen und fertigen Produkten in der Gewinn- und Verlustrechnung $\int$

(de.c.5.91)

[14] $\int[$ [... Grenzkosten $] \mid$ der Finanzmittelbeschaffung $\mid-\int$

[15] $\int\left[\right.$... Unternehmen] | - |, das an falscher Stelle spart $\int$

[16] $\int[\ldots G e f a h r]|-|$, daß es einem bundesweiten Bürgerbündnis genauso ergeht wie der Deutschen Sozialen Union (DSU)

[17] $\int[$...Versuch $]|-|$, den Systemansatz auch in der deutschsprachigen Betriebswirtschaftslehre nutzbar zu machen $\int$ 
Die hier präsentierte Feldstruktur weicht in dem Sinne von anderen ab, ${ }^{7}$ daß hier ein Zusatzfeld angenommen wird. Schon 1971 wies Sommerfeldt die nachgestellten Attribute der Substantivphrase zwei verschiedenen Feldern zu, und zwar dem Nachfeld und dem Zusatzfeld, wobei jedoch die zugrundeliegenden Kriterien nicht explizitiert wurden, und die Abgrenzung nur durch die Unterscheidung zwischen sogenannten "nachgestellten, aber nicht isolierten" und "nachgestellten isolierten Attributen" erfolgte (Sommerfeldt 1971: 19). Schmidt weist darauf hin, daß dieser Vorschlag nicht von anderen Autoren aufgegriffen wurde, und begründet dies damit, daß "die Abgrenzung [...] zu undeutlich bleibt und die entsprechenden Beispiele keine überzeugenden Stellungsgemeinsamkeiten aufweisen" (Schmidt 1993: 92). Meines Erachtens ist es schon möglich, die nachgefragte deutliche Abgrenzung zu machen und die nachgefragten "überzeugenden Stellungsgemeinsamkeiten" zu finden, und zwar wie folgt: Die Unterscheidung zwischen SF und $\mathrm{ZF}$ läßt sich damit begründen, daß die konstituierenden Elemente des ZF im Gegensatz zu den konstituierenden Elementen des SF keine obligatorische Adjazenz verlangen und sich somit aus der Phrase extrahieren lassen (vgl. Beispiel 18 ggü. 19). Aus dem Grunde werden übrigens für die Substantivphrase auch zwei Extraktionsfelder für extrahierbare Elemente der Substantivphrase angenommen. ${ }^{8}$ Es kommen dabei sowohl ein vorangestelltes als auch ein nachgestelltes Extraktionsfeld in Frage, wie aus Abb. $5 \mathrm{zu}$ ersehen ist, in der die gesamte Feldstruktur der Substantivphrase zu sehen ist.

[18] (heute würde ich) $\int$ [ihren $|-|$ Einflu $\left.\beta\right] \mid$ auf den Produktionsablauf $\mid$ -

$\int$ (bezweifeln)

vs.

*(heute würde ich) $\int[$ ihren $|-|$ Einflu $\beta]|-|-\int$ (bezweifeln) $\mid$ auf den Produktionsablauf $\mid$

[19] (wir haben) $\int[$ die $|-|$ Brücke] $|-|$, die ebenfalls im Mittelalter gebaut wurde, $\int$ (besichtigt)

(Nach Weinrich 1993: 360)

vs.

(wir haben) $\int[$ die $|-|$ Brücke $]|-|-\int$ (besichtigt) $\mid$, die ebenfalls im Mittelalter gebaut wurde

(Weinrich 1993: 360)

7 Es sei hier auf beispielsweise Duden-Grammatik 1995, Askedal 1986 und Lauridsen/Poulsen 1995 verwiesen.

8 Es dreht sich um Fälle wie eben in Beispiel 19 oder wie in | auf die Frage | (ist es) $\int$ [keine $|-|$ Antwort $]|-|-\int$. 
Abb. 5

$$
\mid \text { Extr.F }\left|\ldots \int[\mathrm{AF}|\mathrm{VF}| \mathrm{KF}]\right| \mathrm{SF}\left|\mathrm{ZF} \int \ldots\right| \text { Extr.F }
$$

Im Schlußfeld sind übrigens auch solche attributive Substantivrahmen, die als sogenannte enge Appositionen (Beispiel 20) bzw. als eine Art adverbielle Angabe (Beispiel 21) fungieren, sowie gewisse als- / wieKonstruktionen (Beispiel 22), nachgestellte unflektierte Adjektive (Beispiel 23) und schließlich auch Adverbien (Beispiel 24) zu finden.

[20] $\int\left[\right.$ [ein $|-|$ Glas] $\mid$ Wein $\mid-\int$

[21] $\int\left[\right.$ die $|-|$ Sitzung] $\mid$ nächste Woche $\mid-\int$

[22] $\int\left[\right.$ [seine $|-|$ Erfolge] $\mid \underline{\text { als FußBballspieler } \mid-\int}$

[23] $\int[-|-|$ Whiskey $] \mid$ pur $\mid-\int$

[24] $\int[$ der $|-|$ Student $] \mid \underline{\text { dort } \mid-\int}$

Für das VF, das SF und auch für das ZF ist es typisch, daß sie aus mehreren sowohl koordinierten als auch subordinierten Elementen bestehen. Aber auch im AF und im KF befinden sich unter Umständen mehrere Elemente, wie es aus den Beispielen 25 und 26 hervorgeht.

[25] $\int$ [alle diese $|-|$ Fragen] $|-|-\int$

(Helbig/Buscha 1996: 356)

[26] $\int[$ die $|-|$ Einhaltung bzw. Schaffung] $\mid$ bestimmter Relationen zwischen Beteiligungs- und Kreditkapital $\mid$ - $\int$

(de.c.3.30)

\subsection{Das Kettenprinzip}

Das vierte und letzte übergeordnete Prinzip ist das Kettenprinzip, das als das rezeptive Prinzip des Modells und deswegen als das charakteristichste Prinzip bezeichnet werden kann. Den Ausgangspunkt für das Prinzip, das von den prototypischen Attributen des SF begründet ist und die Annahme der Nominalklammer voraussetzt, bildet die Rücksicht auf die Textoberfläche und somit die rezeptive Perspektive, wobei die Elemente der Textoberfläche als Signale für die Rezeption betrachtet werden. In dem Zusammenhang spielt es eine große Rolle, daß die prototypischen Attribute im Schlußfeld (die substantivischen Attribute) morpho-syntaktisch gesehen und im Gegensatz zu den prototypischen Attributen im Vorfeld (die Adjektiv- und Partizipialattribute) dem Kern indirekt angefügt werden in dem Sinne, daß sie nicht mit dem Kern hinsichtlich Numerus, Genus und Kasus kongruieren, sondern dem Kern entweder durch Kasus oder eine Präposition angefügt werden. Von der Form her unterscheidet sich der attributive Substantivrahmen 
also nicht von dem Substantivrahmen, der als Satzglied fungiert. Dies ist übrigens als der wesentlichste Grund dafür zu sehen, daß es dem Rezipienten oft Schwierigkeiten bereitet, zwischen einem Substantivrahmen als Satzglied und als Attribut zu unterscheiden. In einer Arbeit, in der die rezeptive Perspektive einzuarbeiten ist, sollte dies auch berücksichtigt werden - und wird auch eben durch das Kettenprinzip berücksichtigt.

Dem Kettenprinzip liegt die Ansicht zugrunde, daß die konstituierenden Elemente des Schlußfeldes aus Substantivrahmen bestehen, also aus eigenen AF, VF und KF. Diese Ansicht ist auch bei FabriciusHansen/Ahlgren 1986: Kap. 4, sowie bei Fabricius-Hansen/Solfjeld $1994 \mathrm{zu}$ finden. So heißt es hier:

"Ein substantivisches Attribut [...] bildet einen sog. Substantivrahmen [...], insofern es den pränuklearen und nuklearen Teil einer Substantivphrase umfaßt, etwaige postnukleare Teile jedoch ausschließt."

(Fabricius-Hansen/Solfjeld 1994: 49)

Kommen mehrere Attribute dieser Art vor, können auf diese Weise ganze Ketten von Substantivrahmen gebildet werden, und wir sprechen in dem Fall von Attributketten (daher: Kettenprinzip). In den untenstehenden Beispielen 27 und 28 kommen somit viergliedrige Ketten vor.

Das Prinzip läßt sich graphisch wie in Abb. 6 darstellen (vgl. Beispiel 27 und 28).

Abb. 6

$$
\int[\ldots \mathrm{K}]\left|\left[\mathrm{SR}_{1}\right]\left[\mathrm{SR}_{2}\right] \ldots\left[\mathrm{SR}_{\mathrm{n}}\right]\right| \ldots \int
$$

[27] in $\int[$... Form] $\mid$ [der Ermittlung] [des Erwartungswertes] [für die Rentabilität] [des Beteiligungskapitals] | ... J (nach de.c.3.90)

[28] J [... 35-Prozent-Beteiligung]|[des Marktführers] [Ruhrgas AG] [am DDR-Monopolisten] [Verbundnetz,Gas]|... J

(nach de.d.2.32)

Durch das Kettenprinzip wird sehr deutlich, daß sich das rezeptionsgrammatische Modell als ein lineares Modell charakterisieren läßt, so wie schon in Abs. 1 vorgeführt wurde. Im rezeptionsgrammatischen Modell wird somit der Linearisierung grundsätzlich mehr Wert zugeschrieben, als dies sowohl in der traditionelleren als auch innerhalb der generativen Grammatik der Fall ist, so lassen sich die beiden SF der Beispiele 27 und 28 in erster Linie als viergliedrige Attributketten be- 
schreiben, obwohl die zugrundeliegenden syntaktischen Strukturen der beiden SF völlig unterschiedlich aussehen, wie aus Abb. 7 zu ersehen ist.

Abb. 7

Beispiel 27

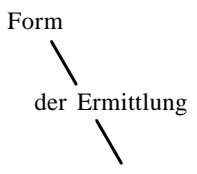

des Erwartungswertes

\}

für die Rentabilität

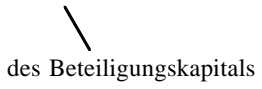

Beispiel 28

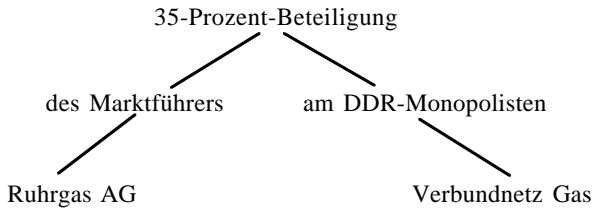

Es ist wichtig, an dieser Stelle zu betonen, daß durch die Beschreibung anhand des rezeptionsgrammatischen Modells keineswegs auf eine Beschreibung der hierarchischen Strukturen im SF verzichtet wird. Nur erfolgt diese Beschreibung erst im Anschluß an eine liniarisierte Beschreibung.

[29] ... Hebelwirkung der Substitution von Beteiligungskapital durch Kreditkapital

Zur Verdeutlichung des Analyseprinzips sei Beispiel 29 angeführt, das traditionell wie folgt analysiert werden würde: Es gibt eine Substantivphrase mit einem nachgestellten substantivischen Attribut ("der Substitution von Beteiligungskapital durch Kreditkapital"), das aus dem Kern ("Substitution") und zwei gleichgeordneten nachgestellten substantivischen Attributen in Form von PP ("von Beteiligungskapital" und "durch Kreditkapital") besteht. Die Struktur ist aus Abb. 8 zu ersehen. 
Abb. 8

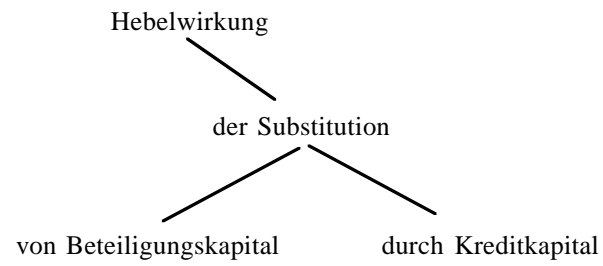

Auf der Basis des rezeptionsgrammatischen Modells ist der substantivische Kern (Hebelwirkung) um drei nachgestellte substantivische Attribute in Form von Substantivrahmen (hiernach: SR) (der Substitution $\left(\mathrm{SR}_{1}\right),{ }^{9}$ von Beteiligungskapital $\left(\mathrm{SR}_{2}\right)$ und durch Kreditkapital $\left.\left(\mathrm{SR}_{3}\right)\right)$ erweitert, wie es aus Beispiel 29.a ersichtlich wird, dabei ist $\mathrm{SR}_{1}$ dem Kern direkt subordiniert, $\mathrm{SR}_{2}$ und $\mathrm{SR}_{3}$ dem Kern indirekt und $\mathrm{SR}_{1}$ direkt subordiniert, was für das SF der Substantivphrase mit anderen Worten genau die hierarchische Struktur ergibt, wie in Abb. 8 zur Unterstützung der traditionellen Beschreibung gezeigt wurde.

[29.a] $\int$ [... Hebelwirkung] | [der Substitution] [von Beteiligungskapital] [durch Kreditkapital] | - J
$\left[\mathrm{SR}_{1}\right]$
$\left[\mathrm{SR}_{2}\right]$
[SR $\left.\mathrm{SR}_{3}\right]$

(de.c.3.70)

Man könnte sich an dieser Stelle zu recht die Frage stellen, warum das Kettenprinzip nur für die Beschreibung des SF und nicht für die Beschreibung des VF herangezogen wird. Hier soll noch einmal auf den prinzipiellen Unterschied zwischen den prototypischen Attributen des VF und des SF erinnert werden, daß die prototypischen Attribute des SF dem Kern indirekt angefügt werden und die prototypischen Attribute des VF direkt in dem Sinne, daß sie mit dem Kern hinsichtlich Numerus, Genus, und Kasus kongruieren. Die Subordination der prototypischen Attribute wird also im VF im Gegensatz zu den prototypischen Attributen im SF morphologisch gekennzeichnet (Beispiel 30 und 31).

[30] $\int\left[\right.$ [ein $\mid$ hübscher $\mid$ Mann] $\mid$ mit braunen Augen $\mid-\int$

[31] $\int$ [eine $|\underline{\text { hübsche }}|$ Frau] $\mid \underline{\text { mit braunen Augen } \mid-\int}$

9 Die Ziffer bezieht sich auf die topologische Stellung des SR. So wird der erste SR im $\mathrm{SF}$ nach dem Kern, von links nach rechts gezählt, $\mathrm{SR}_{1}$ genannt, der zweite $\mathrm{SR}_{2}$ etc. 
Es gibt somit für die Rezeption mit anderen Worten Signale der Subordination im VF. Gleichzeitig soll betont werden, daß das rezeptionsgrammatische Modell grundsätzlich ein Modell zur syntaktischen Beschreibung ist. Die rezeptive Perspektive wird nur an Stellen sichtbar, an denen sie durch oberflächenmäßige Erscheinungen unterstützt werden kann. Das rezeptionsgrammatische Modell ist somit unter Berücksichtigung der rezeptiven Perspektive entstanden, trägt jedoch keineswegs zur Beschreibung der kognitiven Prozesse oder des Verarbeitungsprozesses bei.

Wie aus obenstehendem ersichtlich ist, dürfte das Prinzip grundsätzlich nur für einen Teil der möglichen Attribute im Schlußfeld gelten, zumal sich substantivische Attribute selbstverständlich als SR beschreiben lassen, die übrigen möglichen Attributformen im Schlußfeld, nachgestellte unflektierte Adjektive (Beispiel 23) und Adverbien (Beispiel 24), dagegen jedoch nicht. Trotz diesem grundsätzlichen Unterschied zwischen den möglichen Attributformen im Schlußfeld, wird das Prinzip aus dem Grunde auch auf die eher nicht-prototypischen Attributformen übertragen und somit als übergeordnet betrachtet, weil dem Prinzip als Ausdruck der rezeptiven Perspektive viel Wert beigemessen wird. Daß die nicht-prototypischen Attributformen sich auch als Teil einer Kette betrachten lassen, geht aus Beispiel 32 hervorgeht, in dem $\mathrm{SR}_{1}$ und $\mathrm{SR}_{3}$ prototypische Attribute sind, $\mathrm{SR}_{2}$ und $\mathrm{SR}_{4}$ dagegen nichtprototypische.

[32] in $\int[$ der $|-| H o ̈ h e] \mid[$ der insgesamt ausgewiesenen Erträge] [einerseits] sowie [der Aufwendungen] [andererseits] | - J

(de.c.5.81)

\subsection{Ein Überblick}

In Abb. 9 findet sich das rezeptionsgrammatische Modell mit den vier grundlegenden Prinzipien, dem Phrasen-, dem Klammer-, dem Feldund dem Kettenprinzip. Das Phrasenprinzip wird durch $\int \ldots \int$ symbolisiert, das Klammerprinzip durch [...], das Feldprinzip durch $|. .$.$| und$ schließlich das Kettenprinzip durch $\left[\mathrm{SR}_{1}\right]\left[\mathrm{SR}_{2}\right] \ldots\left[\mathrm{SR}_{\mathrm{n}}\right]$.

Abb. 9

$\mid$ Extr.F. | .. [ AF $|\mathrm{VF}| \mathrm{KF}]\left|\mathrm{SF}\left(\left[\mathrm{SR}_{1}\right]\left[\mathrm{SR}_{2}\right] \ldots\left[\mathrm{SR}_{\mathrm{n}}\right]\right)\right| \mathrm{ZF} \quad \ldots \mid$ Extr.F. $\mid$ 


\title{
2. Analyse
}

Im obenstehenden Abschnitt wurde das rezeptionsgrammatische Modell zur Beschreibung von SP erstellt und diskutiert. In diesem Abschnitt soll gezeigt werden, daß das Modell auch tatsächlich der Beschreibung dienen kann, indem eine Analyse, die in Ditlevsen 1996 ausführlich behandelt wird, hier in Auszügen präsentiert wird. ${ }^{10}$ Wie schon in der Einleitung erwähnt wurde, ist es Ziel der Analysen gewesen, Hypothesen darüber aufzustellen, inwieweit morpho-syntaktische Strukturen der SP zu rezeptiven Schwierigkeiten der dänischsprachigen Deutschstudenten als der tatsächlichen, aber nicht der intendierten Rezipienten beitragen könnten.

Für die Analysen gilt die Grundannahme für die in FabriciusHansen/Solfjeld 1994 präsentierte Vorstudie bezüglich stilistischer Unterschiede zwischen deutschem und norwegischem Sprachgebrauch, daß Unterschiede des Sprachgebrauchs, was z.B. Satz- und Periodenlänge, relative Häufigkeit von Subjekten im Vorfeld, Nominalisierungsdichte betrifft,

\begin{abstract}
"zumindest mitverantwortlich sind für die [...] produktiven wie rezeptiven Unzulänglichkeiten in der Sprachbeherrschung norwegischer Studenten: Ihrer schriftlichen Produktion legen sie eher norwegische als deutsche stilistische Normen zugrunde; und beim Lesen fällt ihnen die Verarbeitung von Konstruktionen, die eine ihnen aus dem muttersprachlichen Lesen nicht vertraute grammatische Komplexität und Informationsdichte aufweisen, besonders schwer." (Fabricius-Hansen/Solfjeld 1994: 3; Hervorhebung mgd)
\end{abstract}

Dieser Ausgangspunkt hat u.a. folgende Konsequenzen für die Grundlage der hier in Auszügen präsentierten Arbeit (vgl. Ditlevsen 1996):

- $\quad$ Da hier Unterschiede / Kontraste zwischen dem in diesem Fall dänischen und deutschen Sprachgebrauch für die Arbeit zentral sind, müssen die Analysen kontrastiv sein. ${ }^{11}$

10 Die hier vorgestellten Ergebnisse werden im Vergleich zu Ditlevsen 1996 auch daraufhin getestet, inwieweit sie statistisch signifikant sind oder nicht. In dem Zusammenhang möchte ich mich bei Niels Reinholt Petersen, Universität Kopenhagen, sowie bei Steen Andersen, Ph.D., Wirtschaftsuniversität Aarhus, für ihre unentbehrliche Hilfe bedanken.

11 Auf diesen Aspekt wird in vorliegendem Aufsatz verzichtet. Es ist jedoch trotzdem wichtig, darauf hinzuweisen, zumal der Aspekt nicht nur die Einbeziehung sowohl deutsch- als auch dänischsprachiger Texte voraussetzt, sondern auch weil er einen erheblichen Einfluß auf die ausgewählten Texte hat (vgl. unten). 
Da die Verarbeitung von dem Rezipienten "unbekannten" Konstruktionen dazu beiträgt, einen Text besonders schwer lesbar zu machen ist es angebracht, quantitative Analysen durchzuführen, nach der Devise: je mehr morpho-syntaktische Konstruktionen es in einem gegebenen Text gibt, die einem DaF-Studenten aus der Muttersprache nicht vertraut sind, desto schwerer fällt ihm der Lese- und damit der Verstehensprozeß.

Für die Analyse wird weiter angenommen, daß die Häufigkeit und die Menge der nicht vertrauten Strukturen in einem Text mit den Schwierigkeiten korrelieren, die die DaF-Studenten beim Leseprozeß haben. Diese Annahme gilt nicht nur für die interlingualen sondern auch für die intra-lingualen Analysen, weil die Analysen eben grundsätzlich aus kontrastiver Perspektive durchgeführt worden sind. ${ }^{12}$

Das untersuchte Objekt sind solche Substantivphrasen, "die nicht selber in Substantivphrasen eingebettet sind, sondern als Satzglieder (Kasusglieder) oder als regierte Nominalphrasen in präpositionalen Satzgliedern dienen" (Fabricius-Hansen/Solfjeld 1994: 43) ${ }^{13}$ und die mit mindestens einer Erweiterung (außer einem evtl. Artikelwort) ausgebaut sind (im weiteren SP). Diese insgesamt vier SP in Beispiel 33 ("den vergangenen drei Jahrzehnten", "regelmäßige Überarbeitungen dieses Buches", "der Weiterentwicklung der Betriebswirtschaftslehre in Theorie und Praxis", sowie "der zweite Abschnitt") wurden somit bei der Analyse berücksichtigt; die Substantivphrase "eine Ausnahme" dagegen nicht.

[33] In $\int[$ den $\mid$ vergangenen drei $\mid$ Jahrzehnten $]|-|-\int$ trugen $\int[-\mid$ regelmäßige $\mid$ Überarbeitungen] $\mid$ dieses Buches $\mid-\iint[$ [der $|-|$ Weiterentwicklung $] \mid$ der Betriebswirtschaftslehre in Theorie und Praxis $\mid-\int$ Rechnung. [...] $\int\left[\right.$ Eine $|-|$ Ausnahme] $|-|-\int$ bildet $\int[$ der $\mid$ zweite $\mid$ Abschnitt] $|-|-\int$.

(nach Wöhe 1996: VI)

\footnotetext{
12 Diese Annahmen liegen zugrunde, wenn im folgenden davon gesprochen wird, daß "X rezeptionsgrammatisch gesehen schwieriger zu verarbeiten ist".

13 Die Definition bezieht sich bei Fabricius-Hansen/Solfjeld 1994 auf den Begriff "maximale Substantivphrase", der dem Begriff "minimale Substantivphrase" (entspricht dem SR) gegenübersteht. In diesem Artikel wird aus terminologischen Gründen auf die beiden Begriffe verzichtet.
} 


\subsection{Korpus}

Das Korpus besteht aus Fachtexten der Wirtschaft, genauer gesagt der Volkswirtschaft und der Betriebswirtschaft, die nach der traditionellen Gliederung die beiden Hauptgebiete der Wirtschaftswissenschaften ausmachen (vgl. beispielsweise Wöhe 1996: 28). Die Fachtexte lassen sich verschiedenen Kommunikationssituationen zuordnen. In Anlehnung an Hoffmann 1987 werden somit Fachtexte verschiedener sogenannter vertikaler Schichten untersucht. ${ }^{14}$ Optimal wäre es gewesen, Fachtexte der obersten Schichten (d.h. wissenschaftliche Fachtexte) zu untersuchen, zumal evtl. morpho-syntaktische Unterschiede hier sicherlich am deutlichsten hervortreten würden, und zumal hier die Fachtexte zu finden sind, die für Fachleute die primären (wissenschaftliche Artikel, Berichte verschiedener Arten, wissenschaftliche Arbeiten) und somit auch für die Zielgruppe der Wirtschaftssprachlichen Fakultät der Wirtschaftsuniversität Aarhus (i.e. für zukünftige Übersetzer) interessant sind. Es hat sich jedoch als äußerst problematisch herausgestellt, dänischsprachige Fachtexte solcher Natur zu finden, die sich gleichzeitig mit deutschsprachigen vergleichen ließen. Ein Grund dafür könnte der sein, daß die dänischen Forscher hauptsächlich auf Englisch publizieren.

Die untersuchten Texte gehören zur einen Hälfte der sogenannten vertikalen Schicht $\mathrm{C}$ an, und zur anderen Hälfte der sogenannten vertikalen Schicht D. Als Kriterium für die Einstufung von Texten habe ich nur einen Parameter herangezogen, und zwar den Parameter Kommunikationsteilnehmer (Abb. 10). ${ }^{15}$

\footnotetext{
14 Die Tendenz in den meisten wissenschaftlichen Fachsprachenarbeiten ist es, eher von Textsorten zu sprechen. Die Einteilung nach Hoffmanns Modell scheint hier jedoch angebrachter, weil die Zuordnung der einzelnen Fachtexte/Fachtextsorten in eine größere Struktur es ermöglicht, Annahmen über Fachtexte/Fachtextsorten anderer Schichten zu machen. Somit wird es möglich sein, aufgrund interlingualer Analysen von deutsch- und dänischsprachigen Texten der Schichten C und D interlinguale Analysen anhand allein deutschsprachiger Texten der oberen Schichten A und B durchzuführen.

15 Hoffmann spricht in dem Zusammenhang von den "Kommunikationsträgern" (Hoffmann 1987: 66). Als weitere Parameter für die vertikale Schichtung von Fachsprachen betrachtet Hoffmann die Abstraktionsstufe, die äußere Sprachform und schließlich das Milieu, "in dem [die] Sprachschichten gebracht werden" (Hoffmann 1987: 65).
} 
Abb. 10

\begin{tabular}{||l|l||}
\hline A & Kommunikation primär unter Experten (Theorie) \\
\hline B & $\begin{array}{l}\text { Kommunikation primär zwischen Experten (Theorie) und Experten } \\
\text { (Praxis) }\end{array}$ \\
\hline C & $\begin{array}{l}\text { Kommunikation primär zwischen Experten (Theorie), Experten } \\
\text { (Praxis) und Experten in spe }\end{array}$ \\
\hline D & $\begin{array}{l}\text { Kommunikation primär zwischen spezialisierten Laien } \\
\text { (Fachjournalisten) und Experten (Praxis) }\end{array}$ \\
\hline E & $\begin{array}{l}\text { Kommunikation primär zwischen spezialisierten Laien } \\
\text { (Fachjournalisten) und Laien }\end{array}$ \\
\hline
\end{tabular}

Unter Texten der vertikalen Schicht C verstehe ich somit in Anlehnung an Hoffmann 1987 Texte einer Kommunikationssituation, an der vor allem Experten (Theorie), Experten (Praxis) sowie Experten (in spe) teilnehmen; konkret handelt es sich hier um Texte aus Lehrbüchern innerhalb der Betriebswirtschaftslehre (BWL). Unter Texten der vertikalen Schicht D verstehe ich wieder in Anlehnung an Hoffmann 1987 Texte einer Kommunikationssituation, an der vor allem spezialisierte Laien (i.e. Fachjournalisten) und Experten (Praxis) teilnehmen; konkret handelt es sich hier um Texte aus Wirtschaftsmagazinen genereller Art (Wirtschaftswoche und Børsens Nyhedsmagasin).

Das Korpus ist klein, zumal es nur aus insg. 100 sogenannten Normseiten (NS) ${ }^{16}$ besteht. Es ist exemplarisch, zumal es nach ganz bestimmten Kriterien aufgebaut worden ist, auf die hier nicht näher eingegangen wird, und schließlich ist es kontrastiv, zumal es aus sowohl deutsch- als dänischsprachigen Texten besteht. Aus Abb. 11 geht die Zusammensetzung des Korpus hervor.

Abb. 11

\begin{tabular}{||l|l|l||}
\hline & Deutsch (de) & Dänisch (da) \\
\hline C & de.c.1 - de.c.5 & da.c. 1 - da.c.5 \\
& (Lehrbücher) & (Lehrbücher) \\
& 25 Normseiten & 25 Normseiten \\
\hline $\mathbf{D}$ & de.d.1 - de.d.10 & da.d.1- da.d.10 \\
& (Wirtschaftswoche $)$ & (Børsens Nyhedsmagasin) \\
& 25 Normseiten & 25 Normseiten \\
\hline
\end{tabular}

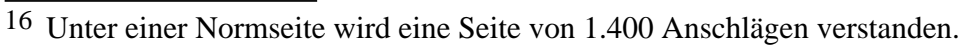


Die untersuchten Texte lassen sich daraufhin als Kombinationen der gezeigten Parameter charakterisieren. Im obersten Kasten links befinden sich somit die deutschsprachigen Texte aus Lehrbüchern der BWL, und im untersten Kasten links die deutschsprachigen Texte aus Wirtschaftsmagazinen genereller Art. Wie aus der Abbildung ersichtlich ist, hat die Zusammensetzung es mir somit erlaubt, sowohl intra- als auch interlinguale Analysen durchzuführen. In diesem Aufsatz wird - wie schon erwähnt - allein auf den intralingualen deutsch-deutschen Analyseteil Rücksicht genommen werden.

\subsection{Intralinguale These und Parameter}

Die zu bestätigende bzw. zu widerlegende intralinguale These sagt nicht überraschend voraus, daß die SP der Schicht C rezeptionsgrammatisch gesehen schwieriger zu verarbeiten sind als die SP der Schicht D. ${ }^{17}$ Der Ausgangspunkt für diese These ist eine Kommunikationssituation, in der die Rezipienten die tatsächlichen, aber nicht die intendierten Rezipienten sind.

Die wichtigsten Parameter lassen sich in vier übergeordnete Kategorien gliedern, und zwar Ausnutzungsgrad, Breite, Tiefe und schließlich Material. Die ersten drei Kategorien sind alle syntaktischer Art, wogegen die Kategorie Material sowohl morpho-syntaktischer als auch lexikalischer Art ist, wie aus untenstehendem zu ersehen ist. Der Ausnutzungsgrad bezieht sich darauf, welche Felder der jeweiligen zu analysierenden SP (abgesehen vom KF) ausgefüllt sind. Anhand der restlichen Parameter wird daraufhin untersucht, wie die Felder gegebenenfalls ausgefüllt sind. Der Parameter Breite (von manchen auch Länge genannt) wird allein für die Beschreibung des VF und des SF verwendet. Im VF macht die Anzahl der Attribute 1. Grades die Breite aus (Beispiel 34 mit zwei vorangestellten Attributen 1. Grades), und im SF die Anzahl der SR (Beispiel 35 mit vier nachgestellten SR ${ }^{18}$ ), die dem Kern sowohl direkt als auch indirekt subordiniert sein können. ${ }^{19}$

$\overline{17}$ Es sei hier nochmals an die unter Abs. 2 vorgestellten Annahmen erinnert, die sich hinter dem Ausdruck verbergen.

18 Zur hierarchischen Struktur des Beispiels, s. Abs. 1.4, Abb. 7.

19 Die unterschiedliche Definition von Breite für das VF und SF ist auf den unter Abs. 1.4 beschriebenen Unterschied zwischen den prototypischen Attributen der beiden Felder und die sich daraus ergebende unterschiedliche Beschreibungsform zurückzuführen. 
[34] J [eine $\mid$ weithin gebräuchliche gedankliche $\mid$ Aufteilung]|des betrieblichen Handlungsprozesses $\mid-\int$

(de.c.2.20)

[35] $\int[$ [die |-|35-Prozent-Beteiligung] des westdeutschen Marktführers Ruhrgas AG am früheren DDR-Monopolisten Verbundnetz Gas $\mid$ - $\int$ (de.d.2.32)

Durch den Parameter Tiefe, der auch allein für die Beschreibung des VF und des SF verwendet wird, werden hypotaktische Strukturen der Substantivphrasen ermittelt. Im VF machen die dem Kern indirekt subordinierten Attribute, i.e. Attribute 2., 3. etc. Grades, die Tiefe aus. Für das Beispiel 36 wurden im VF somit ein Attribut 1. Grades ("eingeführte") und 1 Attribut 2. Grades ("neu") registriert, und für das Beispiel 37 ein Attribut 1. Grades ("ansetzbaren"), ein Attribut 2. Grades ("aufgrund gesetzlicher Bestimmung") und ein Attribut 3. Grades ("gesetzlicher"). Im SF wird die Tiefe durch zwei Kategorien beschrieben. Die erste Kategorie nimmt den Ausgangspunkt bei der hypotaktischen Stellung der SR, so werden für das Beispiel $38 \mathrm{SR}_{1}$ ("auf dem Weg") als Attribut 1. Grades und $\mathrm{SR}_{2}$ ("zu einer europäischen Geldpolitik") als Attribut 2. Grades registriert und für das Beispiel 39 $\mathrm{SR}_{1}$ ("der stark realitätsbezogenen institutionalen Analyse") als Attribut 1. Grades.

[36] $\int\left[\right.$ dieses $\mid$ neu eingeführte $\mid$ Wahlrecht] $|-|-\int$

(de.c.5.27)

[37] $\int\left[\right.$ [den $\mid$ aufgrund gesetzlicher Bestimmung ansetzbaren $\mid$ Größen] $|-|-\int$

(de.c.4.99)

[38] $\int[$ [ein $\mid$ großes $\mid$ Hindernis] $\mid$ auf dem Weg zu einer europäischen Geldpolitik | -

[39] $\int[$ [eine $|-|$ Vorstufe] $\mid$ der stark realitätsbezogenen institutionalen Analyse | - $\int$

(de.c.2.88)

Die zweite Kategorie bezieht sich auf die Attribute in den Vorfeldern der SR. Da die Attribute ausschließlich in Relation zu dem Kern der SR gesetzt werden, und nicht zu dem Kern der übergeordneten SP, lassen sich die Attribute nicht als "Attribute 2., 3. etc. Grades" beschreiben. Für die Beschreibung sind aus pragmatischen Gründen die Begriffe “Attribute '1. Grades', '2. Grades'“etc. benutzt worden. Unter "Attributen '1. Grad' " sind dabei diejenigen vorangestellten Attribute der SR zu verstehen, die dem Kern der SR direkt subordiniert sind, und unter "Attributen '2. Grad'/'2.Gr.'" diejenigen, die einem Attribut '1. Grades' direkt und somit dem Kern der SR indirekt subordiniert sind. Im 
Beispiel 38 wird für $\mathrm{SR}_{2}$ ein Attribut '1. Grades' ("europäischen”) registriert, und im Beispiel 39 werden für SR $_{1}$ zwei Attribute '1. Grades' (“(stark) realitätsbezogenen" und "institutionalen") und ein Attribut '2. Grades' ("stark") registriert.

Der letzte Parameter Material, der wie erwähnt sowohl morphosyntaktischer als auch lexikalischer Art sein kann, gliedert sich wiederum eben in morpho-syntaktisches und lexikalisches Material. Zum morpho-syntaktischen Material, das allein der Beschreibung der vorkommenden Attribute des SF dient, gehören die Kategorien "SR im Genitiv"20 (Beispiel 40) und "SR im Präpositionalkasus" (Beispiel 41) als die beiden wichtigsten, und darüberhinaus auch die Kategorie "Sonstiges", die u.a. sonstige SR (Beispiel 42), "als"-/"wie"-Konstruktionen (Beispiel 43) und Adverbien beinhaltet.

[40] $\int\left[\right.$ kein $|-|$ Einfluß] $\mid$ auf die Höhe des Periodenerfolgs $\mid-\int$

(de.c.5.79)

[41] $\int[$ der $|-|$ Strom $] \mid$ in den alten Bundesländern $\mid-\int$

[42] $\int[$ [die $\mid$ vier $\mid$ Geschäftsbereiche] $\mid$ Luftfahrt, Raumfahrt, Verteidigung und Antriebe | - $\int$

[43] $\int[$ die $|-|$ Auffassung] $\mid$ der Unternehmung als "offenes soziotechnisches System" | -

Für die morpho-syntaktische Beschreibung des Vorfeldes sowohl der SP als auch der SR sind die Kategorien "Partizipialattribute" (Beispiel 44 und 45) und "vorangestellte Substantive im Genitiv" (Beispiel 46 und 47) gebildet worden.

[44] J [die | von den Konzernen auf zunächst 60 Milliarden Mark veranschlagten | Investitionen] | - | - |

[45] $\int\left[\right.$ [ein $|-|$ Yen] $\mid$ über der kalkulierten 115-Dollar/Yen-Parität $\mid-\int$

(de.d.3.4)

[46] $\int[$ GroßBbritanniens $\mid$ staatlichen $\mid$ Speicher $]|-|-\int$

[47] aus $\int\left[\right.$ seiner $|-|$ Zeit] $\mid$ als Kohls Gipfelsherpa $\mid-\int$

Zum lexikalischen Material, das zum VF, KF und SF in Bezug gesetzt wird, gehören in bezug auf das KF und das SF die Kategorien "Komposita" (Beispiel 48 und 49), "Wortgruppen" (Beispiel 50 und 51) und

20 Vorangestellte Substantive im Genitiv wie in $\int[$ die $|-|$ Kunden $] \mid$ in Deutschlands größtem Warenhaus | - $\int$ werden nicht dazu gezählt. Prinzipiell gehören sie zum AF des nachstehenden SR, in der Datenbank wurden sie jedoch fälschlicherweise dem VF zugewiesen. 
"Nomina actionis" (Beispiel 52 und 53), in bezug auf das SF auch die Kategorie "Proprien" (Beispiel 54).

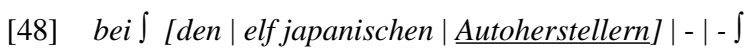

[49] durch $\int[$ den $|-|$ Verkauf] $\mid$ etwa des ostdeutschen Schiffbaus oder der DDR-Versicherung an die westdeutschen Marktführer |- $\quad$ (de.d.2.26)

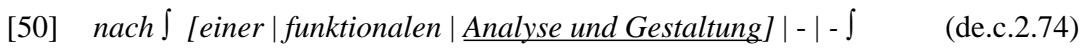

[51] auf $\int[-|-|$ Basis $] \mid$ der Grund- und Anderkosten $\mid-\int \quad$ (de.c.4.46)

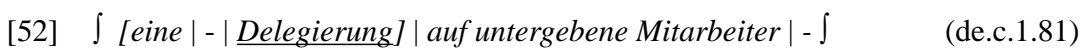

[53] $\int[-|-|$ Gespräche $] \mid$ von Bildschirm zu Bildschirm mit reibungslosem Einspielen von Dokumenten $\mid-\int$

[54] $\int[$ der $|-|$ Strategieexperte $] \mid$ der Dr. August Oetker KG in Bielefeld $\mid-\int$

Von den Parametern, die in Abb. 12 im Überblick präsentiert werden, gelten einige in rezeptionsgrammatischer Hinsicht als besonders rezeptionserschwerend, weil für sämtliche Elemente anzunehmen ist, daß sie dem Rezipienten den Leseprozeß relativ erschweren. Es sei nochmals daran erinnert, daß der Rezipient in diesem Zusammenhang als der Deutschstudent zu verstehen ist, der der tatsächliche, aber nicht der intendierte Rezipient ist. Es wird also keineswegs behauptet, daß die Elemente dem Fachmann Schwierigkeiten bereiten würden. Zu den rezeptionserschwerenden Elementen zählen (in Abbildung 12 fettgedruckt):

- Ausbau nach links (Attribute 2., 3. etc. Grades im VF sowie Attribute im Vorfeld der SR),

- SR im Genitiv,

- Komposita und Nomina actionis im KF und im SF,

- Partizipialattribute im Vorfeld der SP sowie der SR im SF. 
Abb. 12

\begin{tabular}{|c|c|c|c|}
\hline \multicolumn{2}{|c|}{ Parameter } & Kategorien & $\begin{array}{l}\text { wird in Bezug } \\
\text { gesetzt zu }\end{array}$ \\
\hline \multicolumn{2}{|c|}{ Ausnutzungsgrad } & Welche Felder der max. SP sind ausgefüllt? & $\begin{array}{l}\text { VF } \\
\text { SF } \\
\text { ZF / extr.F. } 21\end{array}$ \\
\hline \multirow{2}{*}{\multicolumn{2}{|c|}{ Breite }} & Attribute 1 . Grades & VF \\
\hline & & SR & SF \\
\hline \multirow{2}{*}{\multicolumn{2}{|c|}{ Tiefe }} & Attribute 2., 3. etc. Grades & VF \\
\hline & & $\begin{array}{l}\text { Grad der SR } \\
\text { Attribute im VF der SR }\end{array}$ & SF \\
\hline \multirow[t]{4}{*}{ Material } & \multirow[t]{2}{*}{$\begin{array}{l}\text { morpho- } \\
\text { syntaktisches }\end{array}$} & $\begin{array}{l}\text { SR im Genitiv } \\
\text { SR im Präpositionalkasus } \\
\text { Sonstiges }\end{array}$ & SF \\
\hline & & $\begin{array}{l}\text { Partizipialattribute } \\
\text { vorangestellte Substantive im Genitiv }\end{array}$ & $\begin{array}{l}\text { VF } \\
\text { SF }\end{array}$ \\
\hline & \multirow[t]{2}{*}{ lexikalisches } & $\begin{array}{l}\text { Komposita } \\
\text { Wortgruppen } \\
\text { Nomina Actionis } \\
\end{array}$ & $\begin{array}{l}\mathrm{KF} \\
\mathrm{SF}\end{array}$ \\
\hline & & Proprien & SF \\
\hline
\end{tabular}

Der Großteil der Analysen behandelt die einzelnen Felder im allgemeinen. Darüber hinaus werden auch die tatsächlich vorkommenden SPTypen ermittelt. Für die Kategorisierung der SP-Typen dient der Parameter Ausnutzungsgrad, wodurch die in Abb. 13 präsentierten Kategorien entstehen können. Die SP-Typen sind im übrigen nur bezüglich des Parameters Breite weiter beschrieben worden.

21 Wenn im folgenden auf das Vorkommen von Attributen eingegangen wird, die diesen beiden Feldern zugeschrieben werden, wird nicht zwischen dem Vorkommen im ZF und dem Vorkommen im Extraktionsfeld unterschieden. Die betreffenden Attribute werden konsequent dem ZF zugeschrieben. 
Abb. 13

\begin{tabular}{|c|c|c|}
\hline SP-Typ & Definition & Beispiel \\
\hline $\mathrm{SP}_{\mathrm{VF}}$ & Eine SP nur mit einem ausgefüllten VF & $\begin{array}{l}\text { [die } \mid \text { dadurcherzielbare } \mid \text { Gesamtkapitalrentabilität] }|-| \\
-\quad(\text { de.c.3.59) }\end{array}$ \\
\hline $\mathrm{SP}_{\mathrm{SF}}$ & Eine SP nur mit einem ausgefüllten SF & $\begin{array}{l}\text { [der }|-| \text { Manager }] \mid \text { im Unternehmen der Zukunft } \mid \text { - } \\
\text { (de.d.6.19) }\end{array}$ \\
\hline $\mathrm{SP}_{\mathrm{ZF}}$ & Eine SP nur mit einem ausgefüllten $\mathrm{ZF}$ & $\begin{array}{l}\text { in }[\text { einer }|-| \text { Zeit] }|-|-\ldots \mid, \text { in der die Entscheidungs } \\
\text { situationen immer vielschichtiger und komplizierter } \\
\text { werden } \mid \text { (de.d.1.19) }\end{array}$ \\
\hline $\mathrm{SP}_{\mathrm{VF}+\mathrm{SP}}$ & $\begin{array}{l}\text { Eine SP mit einem ausgefüllten VF und } \\
\text { einem ausgefüllten SF }\end{array}$ & $\begin{array}{l}{[-\mid \text { zeitliche } \mid \text { Beanspruchung }] \mid \text { von Produktionsfak }} \\
\text { toren einer Periode zum Zwecke der Leistungserstellung } \mid \\
\text { - }(\text { de.c. } 4.20)\end{array}$ \\
\hline $\mathrm{SP}_{\mathrm{VF} / \mathrm{SF}+\mathrm{ZF}}$ & $\begin{array}{l}\text { Eine SP mit einem ausgefüllten VF } \\
\text { und/oder einem ausgefüllten SF sowie } \\
\text { einem ausgefüllten } \mathrm{ZF}\end{array}$ & $\begin{array}{l}\text { [die } \mid \text { oberste } \mid \text { Zielsetzung] } \mid \text { der Gesamtunternehmung } \\
\mid \text {, die in der Regel nicht unmittelbar, sondern nur über } \\
\text { Zwischenstufen erreichbar ist } \quad \text { (de.c.1.21) }\end{array}$ \\
\hline
\end{tabular}

\subsection{Auswertung}

Eine erste Analyse der oben im Abs. 2.1 beschriebenen Teilkorpora haben für das deutsche Teilkorpus der Schicht C 473 SP ergeben gegen 495 des deutschen Teilkorpus der Schicht D. Die insgesamt 968 SP, also Substantivphrasen mit mindestens einer Erweiterung ausschließlich Artikelwort, dienen somit als Basis für die Analysen und die Auswertung der Ergebnisse. Von der großen Fülle von untersuchten Teilaspekten werden in diesem Abschnitt nur diejenigen behandelt, die entweder zur generellen Beschreibung der SP beitragen oder besonders interessante Tendenzen andeuten.

\subsubsection{Ausnutzungsgrad}

Aus Tabelle 1 geht hervor, wieviele SP ein ausgefülltes VF, SF bzw. ZF haben. Das VF der SP der Schicht D (im weiteren SP-D) ist signifikant öfter ausgefüllt als das der SP der Schicht C (im weiteren SP-C) (zweiseitiger $\chi^{2}$-Unabhängigkeitstest, pf (Freiheitsgrad) $=1, \mathrm{p}<0,001^{22}$ ), und umgekehrt ist das SF der SP-C signifikant öfter ausgefüllt als das der SP-D (zweiseitiger $\chi^{2}$-Unabhängigkeitstest, $p f=1, p<0,01$ ). Dies wäre auch zu erwarten, zumal das SF innerhalb der SP als Domäne des Nominalstils und das VF als Domäne der Adjektive betrachtet werden kann und zumal für die Texte der Schicht $C$ ein höherer Grad des Nominalstils anzunehmen ist als für die Texte der Schicht D. Das Zusatzfeld wird in beiden Teilkorpora vergleichsweise nur in geringem Umfang

22 Es wird bei den statistischen Angaben wie üblich ein Signifikanzniveau von höchstens $5 \%$ akzeptiert. 
benutzt. Es könnte schon ein bißchen überraschen, daß das Zusatzfeld als Domäne der Verbalisierung als Gegensatz zur Nominalisierung in den SP-D nicht öfter ausgefüllt ist als das der SP-C.

Tabelle 1: Ausnutzungsgrad

\begin{tabular}{||l||l|l||}
\hline \hline Feld & Schicht C & Schicht D \\
\hline \hline VF & $52,64 \%$ & $63,43 \%$ \\
\hline SF & $55,39 \%$ & $45,66 \%$ \\
\hline ZF & $8,46 \%$ & $8,28 \%$ \\
\hline
\end{tabular}

\subsubsection{Breite}

Aus Tabelle 2 ist die Breite des VF bzw. des SF ersichtlich. ${ }^{23}$ Interessant ist es übergeordnet gesehen, daß ung. $90 \%$ der VF sowohl der SP-C als auch der SP-D nur ein Attribut 1. Grades enthalten und ung. 75\% der SF der SP der beiden Teilkorpora nur einen SR. Dies deutet auf eine sehr große Gruppe von syntaktisch gesehen relativ wenig komplexen und wenig umfangreichen SP in den beiden Teilkorpora hin. Wird die jeweilige Breite der beiden Teilkorpora verglichen gibt es hier keinen signifikanten Unterschied zu verzeichnen. Es sei jedoch auf eine mögliche Tendenz hingewiesen. Die SF der SP-C enthalten im Vergleich zu den SP-D öfter, jedoch eben nicht signifikant öfter (zweiseitiger Unabhängigkeitstest, $\mathrm{df}=1, \mathrm{p}<0,10)$ min. drei SR. Inwieweit diese Tendenz stichhaltig ist, müßte an einem umfangreicheren Korpus geprüft werden.

Tabelle 2: Breite

\begin{tabular}{||l|l||l|l||}
\hline \hline Feld & Kategorie & C & D \\
\hline \hline \multirow{3}{*}{ VF } & max. 1 Attribut 1. Grades & $89,96 \%$ & $92,36 \%$ \\
\cline { 2 - 4 } & max. 2 Attributen 1. Grades & $8,84 \%$ & $7,32 \%$ \\
\cline { 2 - 4 } & min. 3 Attributen 1. Grades & $1,20 \%$ & $0,32 \%$ \\
\hline \hline \multirow{3}{*}{ SF } & max. 1 SR & $73,66 \%$ & $76,99 \%$ \\
\cline { 2 - 4 } & $\max$ 2 SR & $17,56 \%$ & $18,14 \%$ \\
\cline { 2 - 4 } & $\max$ 3 SR & $5,34 \%$ & $0,88 \%$ \\
\cline { 2 - 4 } & $\min$ 4 SR & $3,82 \%$ & $3,98 \%$ \\
\hline
\end{tabular}

23 Zur unterschiedlichen Definition der Breite, s. Abs. 2.2. 


\subsubsection{Tiefe}

Wenn es um die VF geht, ist für SP-C ein Attribut 2. Grades in 14,95\% der ausgefüllten VF ermittelt worden gegen $11,80 \%$ für SP-D. In den SP-C sind die ausgefüllten VF somit nicht signifikant öfter (zweiseitiger $\chi^{2}$-Unabhängigkeitstest, $\mathrm{df}=1, \mathrm{p}<0,30$ ) durch ein Attribut 2. Grades erweitert als in den SP-D. Dies gilt auch für das Vorkommen von Attributen 3. Grades, die kaum eine Rolle spielen. In den SP-C kommt ein Attribut 3. Grades somit nur in 1,42\% der ausgefüllten VF vor, in den SP-D nur in 1,77\%. Es kommen in den Teilkorpora keine Attribute 4. Grades vor, und somit sind die VF der SP-C und SP-D gleich tief.

Auf den ersten Blick unterscheiden sich auch nicht die SF der Teilkorpora, was die syntaktische Tiefe betrifft. In sowohl SP-C als auch SP-D sind ung. 86\% der SR 1. Grades. Dies läßt sich nicht nur damit erklären, daß das bloße Vorkommen von SR im SF einen SR 1. Grades auslöst, sondern auch damit, daß - wie oben erwähnt - ein sehr großer Teil der SR im SF nur einen SR enthält und somit nur einen SR 1. Grades. Die Attribute 2. Grades machen in beiden Teilkorpora nur knapp 13\% aus, und die Attribute 3. und 4. Grades spielen kaum, wenn überhaupt, eine Rolle, so kommen in den SF der SP-C nur insgesamt 4 Attribute 3. Grades und 1 Attribut 4. Grades und in den SF der SP-D nur 2 Attribute 3. Grades und keine Attribute 4. Grades vor. Es läßt sich kein signifikanter Unterschied zwischen den beiden Teilkorpora feststellen, auch nicht was die syntaktische Tiefe in Relation zur topologischen Stellung der SR betrifft.

Der letzte Teilaspekt der syntaktischen Tiefe bezieht sich auf den Ausbau nach links der SR im SF, der - wie oben im Abs. 2.2 dargelegt - in der vorliegenden Arbeit zu den rezeptionserschwerenden Elementen gezählt wird. Im Durchschnitt enthält fast jeder dritte SR der SP-C ein vorangestelltes Attribut '1. Grades'24 (30,79\%) gegen nur gut jeden vierten SR der SP-D $(25,66 \%)$, was jedoch kein signifikanter Unterschied ist (zweiseitiger $\chi^{2}$-Unabhängigkeitstest, $\mathrm{df}=1, \mathrm{p}<0,20$ ), sondern nur eine mögliche Tendenz andeutet, daß die durchschnittlichen SP-C in einem größeren Umfang als die entsprechenden SP-D eine Struktur aufweisen und Elemente enthalten, die - relativ gesehen - den Leseprozeß erschweren können. Die Attribute '2. Grades' spielen in den beiden Teilkorpora kaum eine Rolle; sowohl in SP-C als auch in SP-D enthal-

24 Zum Begriff "Attribut '1. Grades"“ etc., s. Abs. 2.2. 
ten nur etwa $2 \%$ der SR ein Attribut '2. Grades', oder anders ausgedrückt nur 6,84\% der Attribute '1. Grades' in SP-C sind um ein Attribut '2. Grades' erweitert gegen 7,69\% in SP-D.

\subsubsection{Material}

Aus Tabelle 3 ist ersichtlich, wieviel Prozent der SR im SF das morphosyntaktische Material GP, PP bzw. "Sonstiges"25 aufweist. Ung. die Hälfte der SR in SP-C kommt als GP vor. Die zweitgrößte Gruppe wird von den PP gebildet, und schließlich gibt es eine kleinere Gruppe von SR, die der Kategorie "Sonstiges" zugewiesen werden. In SP-D bilden die PP die größte, die GP die zweitgrößte und schließlich die Kategorie "Sonstiges" die drittgrößte Gruppe. Die Informationen sind an sich weniger aussagefähig, werden aber durch Vergleich der beiden Teilkorpora und der Verteilung der drei Kategorien interessant. Es fällt u.a. auf, daß die am häufigsten vorkommenden SR der SP-C als GP auftreten und bei den SP-D als PP. Dabei ist der Unterschied zwischen SP-C und SP-D, was das Vorkommen von GP betrifft, signifikant (zweiseitiger $\chi^{2}$-Anpassungstest, $\left.\mathrm{df}=1, \mathrm{p}<0,001\right)$, was das Vorkommen von PP betrifft, jedoch nicht (zweiseitiger $\chi^{2}$-Anpassungstest, $\mathrm{df}=1, \mathrm{p}<0,30$ ). Darüber hinaus fällt es auf, daß die Variationsbreite bei SP-C viel größer ist als bei SP-D $(49,47 \%-12,63 \%=36,84$ Prozentpunkte ggü. $43,42 \%-25,99 \%=17,43$ Prozentpunkte) und schließlich daß die Kategorie "Sonstiges" in SP-D signifikant häufiger vorkommt als in SP-C (zweiseitiger $\chi^{2}$-Anpassungstest, $\mathrm{df}=1, \mathrm{p}<0,001$ ). Dies bedeutet u.a., daß das als rezeptionserschwerend angenommene Element GP in SP-C als texttypisch, vielleicht sogar als Fachtextmerkmal, betrachtet werden kann, daß das häufige Vorkommen der GP in SP-C tatsächlich dazu beitragen kann, den Leseprozeß zu beeinträchtigen, und schließlich, daß das relativ häufige Vorkommen von den Elementen der Kategorie "Sonstiges" in SP-D, die hauptsächlich attributive Appositionen zählt, in die entgegensetzte Richtung weist. Interessant ist übrigens in dem Zusammenhang, was aus der Tabelle nicht zu ersehen ist, daß je weiter nach rechts, d.h. je weiter weg vom Kern der SP, je größer ist die Tendenz, daß die SR der SP-D der syntaktischen Kategorie "Sonstiges" und nicht der Kategorie GP angehören. Gleiches gilt nicht für die SR

25 Zum Inhalt der Kategorie, s. Abs. 2.2. 
der SP-C, was auch ein Indiz für eine komplexere Struktur der SP-C im Vergleich zu den SP-D sein könnte.

Tabelle 3: Morpho-syntaktisches Material im SF

\begin{tabular}{||l||l|l||}
\hline \hline Parameter & Schicht C & Schicht D \\
\hline \hline GP & $49,47 \%$ & $30,59 \%$ \\
\hline PP & $37,89 \%$ & $43,42 \%$ \\
\hline Sonstiges & $12,63 \%$ & $25,99 \%$ \\
\hline
\end{tabular}

Bei der Auswertung des lexikalischen Materials wird an dieser Stelle allein auf die rezeptionserschwerenden Elemente, Kompositum, Nomen Actionis sowie Partizipialattribute, eingegangen. Tabelle 4 ist $\mathrm{zu}$ entnehmen, wieviel Prozent der SP-Kerne im KF ein Kompositum bzw. ein Nomen Actionis ist, wieviel Prozent der SF mindestens ein Kompositum bzw. ein Nomen Actionis ${ }^{26}$ beinhaltet, und schließlich, wieviel Prozent der Attribute 1. Grades im VF und wieviel Prozent der Attribute '1. Grades' im SF ein Partizipialattribut ist. Von zwei Ausnahmen abgesehen (Kern der SP als Kompositum und Attribut '1. Grades' im SF als Partizipialattribut) sind bei sämtlichen rezeptionserschwerenden Elementen signifikante Unterschiede zwischen SP-C und SP-D zu verzeichnen, die alle in dieselbe Richtung weisen (zweiseitiger $\chi^{2}$ Anpassungstest, $\mathrm{df}=1$, Kompositum im SF: $\mathrm{p}<0,01$, Nomen Actionis im KF und im SF, Partizipialattribut im VF: $p<0,001)$ : Es kommt bei SP-C weit öfter ein rezeptionserschwerendes Element vor als bei SP-D und die Häufigkeit, mit der die als rezeptionserschwerend angenommenen morphologischen Elemente in SP-C im Vergleich zu SP-D auftreten, läßt schließen, daß die SP-C zumindest in dieser Hinsicht dazu beitragen, den Leseprozeß zu erschweren.

26 Zu dem Zeitpunkt, als die Datenbank aufgebaut werden mußte, wurden die lexikalischen Parameter nur als zweitrangig betrachtet. Deswegen wurden die lexikalischen Parameter in bezug auf die SR-Kerne nicht direkt auf die einzelnen SR bezogen, sondern eben nur auf das SF als solches. Deswegen ist es u.a. nicht möglich gewesen, evtl. Kombinationen von SR-Kernen, die sowohl als Kompositum als auch Nomen Actionis beschrieben werden können, zu berücksichtigen. Im nachhinein hat sich die eingangs gemachte Disposition jedoch als bedauerlich herausgestellt, zumal die interessantesten Ergebnisse eben bei diesen Parametern zu finden waren. 
Tabelle 4: Rezeptionserschwerendes lexikalisches Material

\begin{tabular}{||l|l||l|l||}
\hline Parameter & Feld & Schicht C & Schicht D \\
\hline \hline \multirow{3}{*}{ Kompositum } & KF & $25,37 \%$ & $29,09 \%$ \\
\cline { 2 - 4 } & SF & $45,80 \%$ & $29,65 \%$ \\
\hline \hline \multirow{2}{*}{ Nomen Actionis } & KF & $23,68 \%$ & $8,28 \%$ \\
\cline { 2 - 4 } & SF & $10,69 \%$ & $1,33 \%$ \\
\hline \hline \multirow{2}{*}{ Partizipialattribut } & VF & $17,27 \%$ & $6,37 \%$ \\
\cline { 2 - 4 } & SF & $13,68 \%$ & $10,26 \%$ \\
\hline
\end{tabular}

Werden die SP-Kerne berücksichtigt, die sowohl als Komposita als auch als Nomina Actionis registriert worden sind, die jedoch nicht in Tabelle 4 auftreten, ergibt sich ein anderes Bild. Von den insgesamt 120 SP-Kerne der SP-C, die als Komposita realisiert sind, sind 8,33\% davon gleichzeitig ein Nomen Actionis, und umgekehrt sind von den insgesamt 112 SP-Kerne der SP-C, die als Nomina Actionis realisiert werden, 8,93\% gleichzeitig ein Kompositum. Wird bei SP-D die Kombination im Verhältnis zu den Komposita gesehen, ergibt sich ein entsprechendes Bild, denn von den insgesamt 144 SP-Kernen der SP-D, die als Komposita realisiert sind, sind 6,94\% davon gleichzeitig ein Nomen Actionis. Interessant ist es aber zu sehen, daß die Kombination 24,39\% der insgesamt 41 SP-Kerne der SP-D, die als Nomina Actionis realisiert werden, ausmacht. Wenn also ein Nomen Actionis als SPKern vorkommt, ist der Kern auch gleichzeitig signifikant öfter ein Kompositum bei SP-D als bei SP-C (zweiseitiger $\chi^{2}$-Unabhängigkeitstest, $\mathrm{df}=1, \mathrm{p}<0,02$ ), was die gestellte These für die Analysen widerspricht. Inwieweit dieser Umstand darauf zurückzuführen ist, daß der Kern der Nomina Actionis bei SP-D öfter als bei SP-C durch ein Valenzglied erweitert worden ist, und inwieweit das gegebenenfalls den Leseprozeß erschwert bzw. erleichtert, müßte weiter untersucht werden.

\subsubsection{SP-Typen}

In den Abschnitten 2.3.1 - 2.3.4 wurden die einzelnen Felder der SP beschrieben ohne Rücksicht auf die gesamte SP, also ohne Rücksicht darauf, inwieweit beispielsweise außer dem VF noch das SF ausgefüllt ist und gegebenenfalls dann wie. In diesem Abschnitt wechselt die 
Perspektive in dem Sinne, daß hier eben die gesamte SP im Mittelpunkt steht und beschrieben werden soll, welche SP-Typen im oben im Abschnitt 2.2 genannten Sinne in welchem Umfang vorkommen. Wie aus Tabelle $5 \mathrm{zu}$ ersehen ist, machen die SP-Typen $\mathrm{SP}_{\mathrm{VF}}$ und $\mathrm{SP}_{\mathrm{SF}}$ in den beiden Teilkorpora insgesamt ung. 80\% der gesamten SP aus und spielen somit die weitaus größte Rolle. Die Bedeutung des VF in den SP-D kommt wie bei der durchschnittlichen SP-D nicht überraschend bei den SP-Typen wieder zum Vorschein, zumal die $\mathrm{SP}_{\mathrm{VF}}$ am häufigsten vorkommen, und signifikant häufiger als in SP-C (zweiseitiger $\chi^{2}$ Anpassungstest, $\mathrm{df}=1, \mathrm{p}<0,05)$. Umgekehrt spielt $\mathrm{SP}_{\mathrm{SF}}$ eine signifikant größere Rolle in SP-C als in SP-D (zweiseitiger $\chi^{2}$-Anpassungstest, $\mathrm{df}=1, \mathrm{p}<0,01)$.

Interessant ist es für beide Teilkorpora, daß die $\mathrm{SP}_{\mathrm{VF}} \mathrm{zu}$ ung. 91\% nur mit einem Attribut 1. Grades erweitert ist, um die $99 \%$ nur mit höchstens zwei, und daß die $\mathrm{SP}_{\mathrm{SF}}$ zu ung. 70-75\% nur mit einem SR erweitert ist, ung. 90\% der SP-C und ung. 94\% der SP-D nur mit höchstens zwei. Alles deutet also darauf hin, daß es in den beiden Teilkorpora eine große Gruppe von SP gibt, die keineswegs als komplex angesehen werden können. Der relativ kleine Anteil der $\mathrm{SP}_{\mathrm{VF}+\mathrm{SF}}$ zeigt dies ebenfalls an. Die beiden SP-Typen $\mathrm{SP}_{\mathrm{ZF}}$ und $\mathrm{SP}_{\mathrm{VF} / \mathrm{SF}+\mathrm{ZF}}$ spielen in den beiden Teilkorpora kaum eine Rolle.

Tabelle 5: SP-Typen

\begin{tabular}{||l||l|l||}
\hline \hline SP-Typ & Schicht C & Schicht D \\
\hline \hline $\mathrm{SP}_{\mathrm{VF}}$ & $38,69 \%$ & $47,88 \%$ \\
\hline $\mathrm{SP}_{\mathrm{SF}}$ & $41,23 \%$ & $31,11 \%$ \\
\hline $\mathrm{SP}_{\mathrm{ZF}}$ & $4,44 \%$ & $4,24 \%$ \\
\hline $\mathrm{SP}_{\mathrm{VF}+\mathrm{SF}}$ & $11,63 \%$ & $12,93 \%$ \\
\hline $\mathrm{SP}_{\mathrm{VF} / \mathrm{SF}+\mathrm{ZF}}$ & $4,02 \%$ & $3,84 \%$ \\
\hline
\end{tabular}

Die $\mathrm{SP}_{\mathrm{VF}+\mathrm{SF}}$ sind in dem Zusammenhang besonders interessant, zumal der weitere Ausbau allem Anschein nach einem bestimmten Muster folgt, was aus der Tabelle nicht ersichtlich ist. So kommen keine Beispiele vor mit sowohl einem VF mit mindestens 2 Attributen 1. Grades als auch einem SF mit mindestens 2 SR. Der weitere Ausbau der $\mathrm{SP}_{\mathrm{VF}+\mathrm{SF}}$ erfolgt also entweder links oder rechts des Kerns der SP. 
Übrigens ist dies noch interessanter aus interlingualer Perspektive, denn im dänischen Korpus erfolgt der weitere Ausbau offensichtlich nicht nach einem solchen Muster.

\section{Konklusion und Perspektivierung}

Ziel der Analysen war es, eine Hypothese darüber aufzustellen, inwieweit morpho-syntaktische Strukturen der Substantivphrase zu den rezeptiven Schwierigkeiten der dänischsprachigen Deutschstudenten beitragen könnten. Zu diesem Zweck wurde ein Modell, das rezeptionsgrammatische Modell, unter gleichzeitiger Berücksichtigung der syntaktischen und der rezeptiven Perspektive erarbeitet. Zu diesem Zweck sind vier grundlegende Prinzipien, das Phrasen-, das Klammer-, das Feld- und schließlich das Kettenprinzip, herangezogen worden, deren Natur voneinander abweichen; so ist nur das Phrasenprinzip als sprachstrukturelles Prinzip zu bezeichnen, die übrigen sind Prinzipien, die als Hilfsmittel zur Beschreibung zu betrachten sind. Hervorzuheben ist dabei das Kettenprinzip, das eine alternative Betrachtungsweise der prototypischen Attribute im SF bietet - eine Betrachtungsweise, die auf die starke Liniarisierung des Modells zurückzuführen ist. Das rezeptionsgrammatische Modell hat Analysen zur Beschreibung der tatsächlich vorkommenden Substantivphrasen aus morpho-syntaktischer Perspektive erlaubt.

$\mathrm{Zu}$ den Analysen aus morpho-syntaktischer Perspektive gehörten die Parameter Ausnutzungsgrad, Breite, Tiefe, morpho-syntaktisches Material. Was diese Parameter betrifft, ließen sich, wenn nicht viele so doch einige, tendenzielle bzw. signifikante Unterschiede zwischen den SP-C und SP-D feststellen. So wird in den SP-C das SF signifikant öfter ausgenutzt als bei den SP-D, bei denen umgekehrt das VF signifikant öfter ausgenutzt wird als bei den SP-C. Das als rezeptionserschwerend betrachtete Element "Vorkommen von GP" kommt bei den SP-C signifikant öfter vor als bei den SP-D; das Element "Ausbau nach links" tendenziell öfter. Die eingangs aufgestellte These, daß die SP-C rezeptionsgrammatisch gesehen schwieriger zu verarbeiten sind als die SPD, läßt sich aufgrund der morpho-syntaktischen Analysen nur teilweise bestätigen. Überraschend ist es übrigens u.a., daß bei den Parametern Breite und Tiefe kein signifikanter Unterschied zu verzeichnen war. 
Durch Einbeziehung der Analyseparameter lexikalischer Art in die Analysen läßt sich die These jedoch durchaus bestätigen. In den SP-C kommen fast ausnahmslos weitaus signifikant mehr Komposita, Nomina Actionis und Partizipialattribute vor als in den SP-D.

Zusammenfassend haben die Analysen zur Beschreibung der SP in Texten aus verschiedenen fachspezifischen Kommunikationssituationen beigetragen, wodurch text(sorten)spezifische Merkmale entstanden sind, die es uns erlauben, bei anderen Textsorten Annahmen als Basis für spezifische Untersuchungen an einem neuen Korpus zu machen. Solche Untersuchungen könnten folgendes als Objekt haben:

\begin{tabular}{||l|l|l||}
\hline Parameter & Element & Zu beantwortende Fragen \\
\hline \hline Tiefe & Ausbau nach links & $\begin{array}{l}\text { Welches Material bildet den Ausbau? } \\
\text { Wie umfangreich ist er? }\end{array}$ \\
\hline Material & Kompositum & $\begin{array}{l}\text { Welche Arten kommen vor? } \\
\text { Wie werden sie attribuiert? }\end{array}$ \\
\hline Breite & SP-Typen & $\begin{array}{l}\text { Inwieweit läßt sich die Tendenz zur Einseitigkeit bei weiterem } \\
\text { Ausbau bestätigen? }\end{array}$ \\
\hline
\end{tabular}

Das relativ häufige Vorkommen von Nomina Actionis und Partizipialattributen bei SP-C deutet auf Deverbalisierung unter Beibehaltung verbalen Inhalts hin. Vor dem Hintergrund wäre es darüber hinaus äuBerst interessant, Analysen aus einer Valenzperspektive durchzuführen. Die Fragen, die in dem Zusammenhang zu beantworten wären, könnten in dem Fall lauten: Inwieweit sind Valenzglieder vorhanden? - und gegebenenfalls welche?

\section{Anhang}

Im Artikel verwendete, nicht übliche Abkürzungen

AF Artikelfeld (= das linke Klammerfeld)

AW Artikelwort

Extr.F. Extraktionsfeld

K Kern

KF $\quad$ Kernfeld (= das rechte Klammerfeld)

SF Schlußfeld

SP Substantivphrasen, "die nicht selber in Substantivphrasen eingebettet sind, sondern als Satzglieder (Kasusglieder) oder als regierte Nominalphrasen in präpositionalen Satzgliedern dienen" (Fabricius-Hansen/Solfjeld 1994: 43) und die mit mindestens einer Erweiterung (außer einem evtl. Artikelwort) ausgebaut sind (= Analyseobjekt) 
SP-C SP der Schicht C

SP-D SP der Schicht D

SR Substantivrahmen

$\mathrm{SR}_{1}$ der erste Substantivrahmen im SF nach dem Kern, von links nach rechts gezählt

VF Vorfeld

ZF Zusatzfeld

\section{Literatur}

Admoni, W.G. (1934). Über die Wortstellung im Deutschen. In Zwei Welten 6. 77-79.

Askedal, John Ole (1986). Zur vergleichenden Stellungsfelderanalyse von Verbalsätzen und nicht-verbalen Satzgliedern (1) / (2). In Deutsch als Fremdsprache 5 bzw. 6. 269-273 bzw. 342-348.

Benes, Eduard (1981). Die formale Struktur der wissenschaftlichen Fachsprachen in syntaktischer Hinsicht. In Bungarten, Theo (Hrsg.) (1981). Wissenschaftssprache. Beiträge zu Methodologie, theoretischer fundierung und Deskription. München: Wilhelm Fink Verlag.185-212.

Bhatt, Christa (1990). Die syntaktische Struktur der Nominalphrase im Deutschen. Tübingen: Narr Verlag.

Bierwisch, Manfred (1961). Ein Modell für die syntaktische Struktur deutscher Nominalgruppen. In ZPSK (Zeitschrift für Phonetik, Sprachwissenschaft und Kommunikationsforschung) 14. 244-278.

Boost, Karl (1964). Neue Untersuchungen zum Wesen und zur Struktur des deutschen Satzes. Berlin: Akademie-Verlag.

Ditlevsen, Marianne Grove (1996). Den udvidede substantivfrase i udvalgte фkonomiske fagtekster - en receptionsgrammatisk analyse i intra- og intersprogligt perspektiv. MS : Wirtschaftsuniversität Aarhus.

Drach, Erich (1940). Grundgedanken der deutschen Satzlehre. Darmstadt: Wissenschaftliche Buchgesellschaft. 1963.

Duden-Grammatik 1995 = Duden. Grammatik der deutschen Gegenwartssprache. Mannheim, Leipzig, Wien, Zürich: Dudenverlag.

Eichinger, L.M. (1992). Vom Nutzen der Nominalklammer. Eine funktionale Erklärung für die Reihenfolge gestufter Adjektivattribute im Deutschen. In Vuillaume et al. (Hrsg) (1992). Die Nominalgruppe im Deutschen (= Eurogermanistik 1 - Europäische Studien zur deutschen Sprache). Tübingen: Gunter Narr Verlag. 86-104.

Eisenberg, Peter (1989). Grundriß der deutschen Grammatik; Stuttgart: Metzler.

Engel, Ulrich (1988-1991). Syntax der deutschen Gegenwartssprache. Heidelberg: Julius Groos Verlag.

Fabricius-Hansen, Cathrine \& Ahlgren, Bengt (1986). A lese tysk sakprosa. En innføring i grammatisk leseteknikk; Oslo, Universitetsforlaget. 
Fabricius-Hansen, Cathrine \& Heringer, Hans-Jürgen (1988). Die Idee einer rezeptiven Grammatik und ihre Realisierung. In Info-DaF 15. 164-175.

Fabricius-Hansen, Cathrine \& Solfjeld, Kåre (1994). Deutsche und norwegische Sachprosa im Vergleich. Ein Arbeitsbericht. (= Arbeitsberichte des Germanistischen Instituts der Universität Oslo $\mathrm{Nr}$. 6). Oslo.

Gallmann, Peter \& Lindauer, Thomas (1994): Funktionale Kategorien in Nominalphrasen. In Beiträge zur Geschichte der Deutschen Sprache und Literatur (PBB) 116/1. 1-27.

Haider, Hubert (1988). Die Struktur der deutschen Nominalphrase. In Zeitschrift für die Sprachwissenschaft 1. 32-59.

Haider, Hubert (1992). Die Struktur der Nominalphrase - Lexikalische und funktionale Strukturen. In Hoffmann, Ludger (1992). Deutsche Syntax - Ansichten und Aussichten. Berlin: Walter de Gruyter. 304-333.

Helbig, Gerhard \& Buscha, Joachim (1996). Deutsche Grammatik. Ein Handbuch für den Ausländerunterricht. Leipzig, Berlin, München, Wien, Zürich, New York: Langenscheidt.

Heringer, Hans Jürgen (1989). Lesen, lehren, lernen: Eine rezeptive Grammatik des Deutschen; Tübingen: Max Niemeyer Verlag.

Hoffmann, Lothar (1987). Kommunikationsmittel Fachsprache. Eine Einführung. Berlin: Akademie-Verlag.

Kolde, G. (1985). Zur Topologie deutscher Substantivgruppen. Rahmenbildung und mehrfache Attribuierung. In Zeitschrift für germanistische Linguistik 13. 241-277.

Kretzenbacher, Heinz Leonhard (1991). Syntax des wissenschaftlichen Fachtextes. In Fachsprache 3-4. 118-137.

Lauridsen, Ole \& Poulsen, Sven-Olaf (1995): Tysk grammatik. København: Munksgaard.

Löbel, Elisabeth (1990): Typologische Aspekte funktionaler Kategorien in der Nominalphrase. In Zeitschrift für Sprachwissenschaft 1/2. 135-169.

Rall, Marlene \& Engel, Ulrich \& Rall, Dietrich (1985): DVG für DaF: DependenzVerb-Grammatik für deutsch als Fremdsprache. Heidelberg: Julius Groos Verlag.

Sommerfeldt, Karl-Ernst (1968). Zur Struktur der Substantivgruppe in einigen funktionalen Stilen. In Deutsch als Fremdsprache 5. 287-295.

Sommerfeldt, Karl-Ernst (1971). Zur Wortstellung in der Gruppe des Substantivs. In Deutsch als Fremdsprache 1. 13-19.

Teubert, Wolfgang (1979): Valenz des Substantivs. Attributive Ergänzungen und Angaben (= Sprache der Gegenwart 49). Düsseldorf: Pädagogischer Verlag Schwann.

Vater, Heinz (1986). Zur NP-Struktur im Deutschen. In Vater, Heinz (Hrsg.) (1986). Zur Syntax der Determinantien. Tübingen: Gunter Narr. 123-145. 
Vater, Heinz (1992). Assymmetrie deutscher Nominalphrasen. In Valentin, Paul (Hrsg.) (1992). Rechts von N. Untersuchungen zur Nominalgruppe im Deutschen. Tübingen: Gunter Narr. 15-26.

Weinrich, Harald (1993). Textgrammatik der deutschen Sprache. Mannheim, Leipzig, Wien, Zürich: Dudenverlag.

Wöhe, Günter (1996). Einführung in die Allgemeine Betriebswirtschaftslehre. München: Franz Vahlen.

Zimmermann, Ilse (1991). Die Syntax der Substantivgruppe: Weiterentwicklungen der X-Theorie. In Ilse Zimmermann (Hrsg) (1991). Syntax und Semantik der Substantivgruppe (= Studia Grammatica 33). Berlin: Akademie-Verlag. 1-32. 
